\title{
MONITORING A COMMON AGENT: implications for financial contracting ${ }^{1}$
}

\author{
Fahad Khalil \\ University of Washington \\ Seattle, USA \\ khalil@u.washington.edu
}

\author{
David Martimort \\ University of Toulouse, IDEI \\ Toulouse, France \\ martimor@cict.fr
}

September 12, 2005

\author{
Bruno Parigi \\ Department of Economics \\ University of Padova, Italy \\ brunomaria.parigi@unipd.it
}

\footnotetext{
${ }^{1}$ We acknowledge helpful comments from Mitchell Berlin, Jacques Crémer, Mathias Dewatripont, Jacques Lawarrée, Chris Papageorgiou, Jean-Charles Rochet, Dick Startz, two referees and an editor, and audiences at the Universities of Freiburg, Lausanne, Michigan State, Mannheim, New Orleans, Oxford, Padova, Southern California, Victoria, Washington, the European University Institute, ECARES, the EARIE Conference 2001, and the Summer Meeting of the Econometric Society 2002. Parigi acknowledges financial support and hospitality from the Finance and Consumption Chair at the European University Institute. The usual disclaimer applies.
} 


\begin{abstract}
Multiple principals want to obtain income from a privately informed agent and design their contracts non-cooperatively. The degree of coordination between principals shapes the contracts and affects the amount of monitoring. Equity-like contracts and excessive monitoring emerge when principals coordinate or verify each others' monitoring efforts. When this is not possible, free riding weakens monitoring incentives, so that flat payments, debt-like contracts and very low levels of monitoring appear. Free riding may be so strong to induce even less monitoring than if the principals cooperated with each other; that is non-cooperative monitoring does not necessarily lead to excessive monitoring.
\end{abstract}

Key words: monitoring; common agency; costly state verification.

JEL classification: D2, D8, G2, G3. 


\section{Introduction}

It is not uncommon to find situations where several principals monitor a common agent: a worker may have multiple supervisors, a bank may have multiple regulators, a firm may borrow from different banks, and individuals are taxed by multiple authorities. Our focus here is on contexts where multiple principals attempt to obtain income from a privately informed agent, such as in the case of multiple financiers. While this problem of costly repayment enforcement has been widely studied in the case of one principal and one agent ${ }^{1}$, little attention has been paid so far to the strategic interactions between multiple principals arising in those environments.

Consider the case of multiple financiers engaged in an income division game with a common agent. In our model, principals can use two instruments to extract the returns of a project. They can monitor the agent and they can demand a transfer from the agent. Monitoring comprises of all the costly instruments aimed at verifying and obtaining income from the agent once it is realized, like certification of balance sheets, supervision, shareholders' legal actions, etc. When coordination problems between principals are not severe so that monitoring can be centralized, there is excessive monitoring compared to when the financiers cooperate, and profit-sharing schemes (or equitylike contracts) are optimal. If coordination problems are severe such that financiers choose their monitoring efforts independently, free riding in monitoring efforts reduces the incentive to monitor and it is optimal not to monitor high profit levels. That is, the repayment is non-contingent on profit when profit levels are high and contracts have debt-like features. Furthermore, free riding may be so strong that there may even be less monitoring compared to when the financiers fully cooperate and merge as one. Therefore, we show that non-cooperative contracting does not necessarily lead to excessive monitoring.

In some environments, monitoring activities are coordinated, while in others they are not. For example, in publicly-traded companies there are many providers of funds and there might also be different classes of security holders, but there is also in place a set of rules and institutions (auditors, bankruptcy courts, disclosure rules) aimed at guaranteeing coordinated access to profits and information to all outside providers of funds. In these environments each principal or financier can demand potentially different transfers from the entrepreneur or agent but there is still a centralized governance mechanism that limits the amount of income an agent can retain by monitoring on behalf of all financiers. On the other hand, situations also abound where no common institution can be constructed to extract the returns of the project. This is the case when the activity is too idiosyncratic and information sensitive (e.g. at the early stages of a company's development). Also, in privately held companies or when the financiers provide large amounts of funds, each has the incentive and the skills to independently affect the extraction of income both

\footnotetext{
${ }^{1}$ See for example the classic pieces Townsend [39], Gale and Hellwig [13], and Border and Sobel [6].
} 
through monitoring and through the transfers. ${ }^{2}$

To present the intuition behind our main results, we take as a benchmark the merged principals case where the principals can coordinate both monitoring and contracting. Consider then an environment where principals are able to verify each others monitoring efforts, so that monitoring can be coordinated or centralized, but each financier can demand potentially different transfers from the entrepreneur. For instance, each financier may independently arrange for private transfers based on the information revealed by a central monitor. We find that there will be greater monitoring and rent extraction than if the principals merged together. This happens even though monitoring results in a public good - its benefit is shared by the principals. Each principal derives a positive externality from the others' contracts, which negates the public good problem. Roughly, by decreasing the repayment he requests from the agent and relying more on direct monitoring, a given principal attempts to induce other principals to believe that profit is low and to reduce the repayments they respectively ask for.

If the principals do not cooperate in monitoring and choose their efforts independently, we say monitoring is decentralized. In this case, there is free riding in monitoring efforts and consequently, the positive externality from the other principals' contracts is now counteracted by a negative externality since each principal under-provides monitoring effort. We show that, in equilibrium, the second force dominates and we obtain a number of results. Not only there is less monitoring than in the centralized case, but there also exist equilibria which involve less monitoring than even in the merged principals case. Free riding between principals leads to a collapse of monitoring for high profit levels and principals rely instead on constant transfers, which introduce debt-like features in the financial contracts. ${ }^{3}$ This is in contrast to the case of centralized monitoring, where profit sharing contracts are optimal. It is also worth noting that a zero-monitoring region arises in our model because of contractual externalities and not from an assumption about the monitoring technology as in the well-known costly state verification (CSV) approach. In the CSV models, the optimality of debt contracts is typically derived from an assumption of constant marginal cost of verifying income or an arbitrary restriction to deterministic auditing. ${ }^{4}$

The point that equity is more useful in settings where monitoring costs are low has also been noted by Boyd and Smith [7], who show that the optimal mix of debt and equity in a company's liability structure depends on the observability of the returns of the underlying assets. When it is possible to combine investment technologies with differences in observability of the returns, the

\footnotetext{
${ }^{2}$ See for example Schleifer and Vishny [34] and Pagano and Röell [27].

${ }^{3}$ The reduction of monitoring when principals choose efforts independently, may be seen as another example of the parallel that Tirole [37] draws between low-powered incentives in multi-principal problems and in multi-task agency, where it is important not to make payments too sensitive to the performance of a single task to the detriment of attention to other tasks.

${ }^{4}$ See for example Townsend [39], Gale and Hellwig [13], and Freixas and Rochet [12].
} 
technology with unobservable returns is funded with debt while the technology with observable returns leads to equity financing.

Since Diamond [11] it is well known that delegating monitoring activity to a common or centralized monitor avoids duplication when many financiers are involved in financing a project. Our findings, however, challenge the notion that non-cooperative contracting leads to excessive monitoring with respect to the merged principals case. As indicated above, we find that the validity of this claim depends on the type of monitoring arrangement (centralized or decentralized) the non-cooperative financiers follow. Our result that there is less monitoring in the decentralized case than in the centralized case is linked to Carletti [8], where two-bank lending may be preferred to single bank lending because single-bank lending results in excessive monitoring in a moral hazard setting.

The issue of multiple lending in models of costly repayment enforcement has been rarely tackled. The early studies that focused on delegated monitoring following Diamond [11] do not look at the strategic interaction between financiers. A paper closely related to ours is Winton [40] that studies how seniority of creditors reduces the amount of costly auditing. Besides using a different monitoring technology, that paper does not consider the strategic interactions between financiers using a common agency framework as we have done.

Contractual externalities have also been identified to be important in the context of multiple lending. Tirole [38] argues that investors do not have incentive to take into account the impact of the various elements of their lending relationship (level and structure of investment, monitoring, and exercise of control rights) on the returns of the other investors. In a moral hazard setting, Bizer and DeMarzo [3] show that when agents may borrow sequentially from several lenders, the level of indebtedness increases with respect to the case in which the borrower can commit to borrow from one bank only.

In this paper we take as given the presence of multiple financiers, but it is easy to argue why that may be so. A first set of reasons has to do with diversification. For instance the separation of ownership and control in public companies where many investors, often small and dispersed, hire professional managers to run firms raises issues of multiple funding. Diversification is also the reason for multiple sources of funds in syndicated loans, LDCs lending, and when regulations prevent banks from gaining control of firms, or limit the exposure of a financial institution to a single risk. Lenders may simply be resource constrained as in Dewatripont and Maskin [10]. Other reasons are related to information: multiple lending may limit a bank's information rents at an interim stage (Hellwig [16], Rajan [31]), provides protection against information loss following a bank's distress (Detragiache, Garella and Guiso [9]), deters strategic default by making reorganization more costly (Bolton and Sharfstein [5]), and makes the soft-budget-constraint problem less 
severe (Dewatripont and Maskin [10]). Multiple banking relations may also result from the trade off between the costs of effort duplication and sharing of monitoring benefits on the one hand, and the benefit from the diseconomies of scale in monitoring each bank faces when monitoring alone on the other hand (Carletti [8]). Funding may also arise as a by product of another trade. For example, in trade credit, multiple lending occurs when several suppliers provide inputs to the same customer that does not pay cash. Finally, in a model with complete information, Parlour and Rajan [28] show that multiple contracting in an unsecured credit market is an equilibrium outcome when the borrower's default payoff is increasing in the number of loans accepted.

Although cast in the realm of financial contracting, our paper also contributes to the more general theory of common agency under adverse selection. ${ }^{5}$ This literature has analyzed contractual externalities between competing mechanism designers when principals may control different "activities" of a common agent who has private information about some parameter relevant for contracting. In our financial contract framework, these "activities" correspond to the monitoring efforts of each principal and the agent has private information on realized income. At a general level, the literature has stressed the inefficiency in rent extraction that arises in such contexts and how contractual externalities depend on whether the contracting activity controlled by one principal affects directly or not the utility function of competing mechanism designers. ${ }^{6}$ Direct externalities are present in our framework as a financier benefits when others monitor. We show that these externalities play a significant role in explaining the structure of contracts. Understanding how limits on contracting affect outcomes is precisely the task that we undertake below in our financial contracting environment when we compare the contractual outcomes which arise when financiers can verify and contract over the monitoring efforts of others and when they cannot. In that respect, this paper contributes to the literature on incomplete contracting by tracing out inefficiencies and contractual forms as contracts are less and less comprehensive. We show therefore that transaction costs that prevent comprehensive and cooperative contracting have a significant impact on the shapes of observed financial contracts. Equity-like contracts emerge for environments with low transaction costs, i.e., when the principals can verify each other's monitoring efforts, whereas debt-like features appear otherwise.

\footnotetext{
${ }^{5}$ Applications already span several fields. Laffont and Tirole [19] analyze regulation; Martimort ([22] and [23], Mezzetti [26] and Stole [36] deal with non-linear pricing and manufacturer-retailers relationships; Biais, Martimort and Rochet [3] study competing market-makers in financial markets. Martimort and Stole [24] and Peters ([29], [30]) offer theoretical frameworks to understand what are the natural classes of mechanisms to be studied. For models of common agency under moral hazard, see Bernheim and Whinston ([1], [2]) among others.

${ }^{6}$ For instance, Martimort and Stole [25] analyze a common retailer-manufacturers example. See also the more abstract framework of Peters [30] who stresses the role of direct externalities between the principal on the kind of mechanisms which can be used. Segal [33] offers a framework for dealing with contractual externalities under complete information but reverses the role of the principal and the agents.
} 
The paper is organized as follows. In Section 2 we lay out the basic model. In Section 3 we analyze the case where principals cooperate. In Section 4 we move to the case where principals play non-cooperatively in transfers but cooperate in monitoring efforts. In Section 5 we consider the case where principals compete both in transfers and in monitoring. We conclude in Section 6 . Most of the proofs are relegated to the Appendix.

\section{The Model}

An investment project needs two inputs: capital and the service of a specialized agent. The agent has zero wealth so outsiders must provide funds if the project is to be realized. We assume that two principals (financiers) $P_{1}$ and $P_{2}$ who do not have the skills to realize the project provide the agent with funds. Technically, we consider a case of intrinsic common agency, i.e., without funding from both principals, the project would not be realized. ${ }^{7}$ For simplicity, we assume that each principal provides half the funding. The agent's task is to use his specialized skills to realize the project and deliver the realized profit to the principals. The investment of fixed size returns a stochastic profit $\theta$, the state of nature, with $\underline{\theta} \leq \theta \leq \bar{\theta}$ where the lowest bound on profit is $\underline{\theta}>0$. Except when explicitly stated, we will also assume that $\bar{\theta}<\infty$. The parameter $\theta$ has a density function $f(\theta)>0$ for all $\theta$, a cumulative distribution function $F(\theta)$, and, for technical reasons that will be clear later, a weakly decreasing hazard rate $\frac{1-F(\theta)}{f(\theta)}$. The principals and agent are risk neutral.

We consider an environment in which the agent is able to observe the realization of the profit or income, $\theta$, while the principals know only its distribution. This gives the agent the opportunity to keep some of the realized profit instead of delivering all to the principals. Thus, we follow a large literature including both the costly state verification (CSV) models (Townsend [39], Gale and Hellwig [13]), and more broadly recent analyses of how company insiders may try to expropriate outside investors (see e.g., Pagano and Röell [27], La Porta and al. [21], and Schleifer and Wolfenson [35]).

Once profit is realized, each principal $P_{k}$ can devote an effort $p_{k} \in[0,1], k=1,2$, monitoring the agent to verify and recover realized profit. We assume that the monitoring effort made by a principal is observable both by this principal and the agent and can be contracted upon by those two parties. Monitoring with overall effort $q$ makes a fraction $q \in[0,1]$ of realized income $\theta$ verifiable, which the principals can therefore recoup. We will assume that the monitoring technology links the individual monitoring efforts of the principals to the fraction $q$ through the formula $q=Q\left(p_{1}, p_{2}\right)=p_{1}+p_{2}-p_{1} p_{2}$. This fraction $q$ increases thus with the monitoring

\footnotetext{
${ }^{7}$ For example, each financier is resource constrained and can only finance part of the capital needed to implement the project. Dewatripont and Maskin [10] make a similar assumption.
} 
efforts $p_{k}$, and thus monitoring by one principal benefits also the other principal. However the marginal benefit of increasing effort by one principal decreases as the other himself exerts a higher effort. This captures the fact that the actions of the principals may result in wasteful monitoring duplications. ${ }^{8}$ Monitoring effort for principal $P_{k}$ comes at a cost given by the function $C\left(p_{k}\right)$, where $C(0)=0, C^{\prime}\left(p_{k}\right)>0$ for $p_{k}>0, C^{\prime \prime}\left(p_{k}\right)>0$ for all $p_{k}$, and $C(\cdot)$ satisfies the Inada conditions $C^{\prime}(0)=0, C^{\prime}(1)=\infty$.

We therefore model in a stylized fashion one of the central issues of corporate governance: namely that outsiders must spend resources to induce insiders to pay out. Our analysis focuses on the strategic aspects of the use of these resources by non-cooperating principals. In the corporate environment monitoring effort can take various forms. It can represent the use of institutions like lawyers, certified public accountants, auditors, bankruptcy courts, independent directors, and legal actions by shareholders. These actions help verify realized income and recover it for outsiders. The strength of such actions is typically inversely related to performance: in other words, we expect to find that the principals will scrutinize the agent more intensively the less income the agent pretends to have had. Of course, the extent to which these monitoring actions can be pursued depends on the legal environment (La Porta and al. [21]).

To alleviate the need to exert monitoring effort, the principals may also ask for a transfer from the agent. Thus, we assume that the agent pays an amount $t_{k}$ to each principal. If there were full information, each principal would use only $t_{k}$ to obtain their share of the profit. Under asymmetric information however, the principals could get at most $\underline{\theta}$ if they relied on transfers alone. The principals' monitoring efforts not only allow them to directly recover a part of realized profit, but may also allow them to obtain higher transfers, for example, by designing contracts that trigger greater monitoring effort for smaller transfers. Thus, the total fraction of income that accrues to the principals is $\frac{q \theta+t_{1}+t_{2}}{\theta}$, where $q$ represents the direct impact of recovery effort.

The yield from the principals' monitoring efforts, $q \theta$, is divided equally between the principals. Thus a principal $P_{k}$ 's payoff is the transfer from the agent plus half the verified profit minus the cost of the monitoring effort of that principal:

$$
W_{k}=t_{k}+\frac{q \theta}{2}-C\left(p_{k}\right) .
$$

The agent's payoff is the part of realized theta that does not accrue to the principals net of the transfers:

$$
U=(1-q) \theta-t_{1}-t_{2}
$$

The agent is protected by limited liability so that $U \geq 0$ for every profit level, and the opportunity cost for the agent's skills is normalized to zero. Thus, the agent's payoff represents the rent

\footnotetext{
${ }^{8}$ In footnote 20, in Section 5, we discuss the implications if the marginal impact of one principal's effort on $q$ increased with the other principal's effort.
} 
from private information about $\theta$ as limited by the two instruments the principals can employ monitoring and transfers. In reality, this rent is likely to be a combination of explicit and implicit rewards. Executive compensation offers an example of explicit rewards: both the level of executive pay and the importance of performance pay indicate that executive compensation packages are often structured to deal with agents who have an informational advantage. On the contrary, in less sophisticated financial contexts, payments of information rents may also take implicit forms like tunneling, corporate perks, etc., or outright theft. More generally the information rent is a component of the control rights that informed insiders can obtain at the expense of outside investors and that can be found in different degrees in all types of financial systems.

The principals' opportunity cost of funds and the monitoring cost are small enough so that the participation constraint of each principal never binds. Normalizing the principals' opportunity cost of funds and the size of the investment to one, we must thus have the expected value of $W_{k}$ to be greater than $\frac{1}{2}$, a condition that we assume to be satisfied in this analysis, and which necessarily holds when uncertainty on types, $\bar{\theta}-\underline{\theta}$, is small enough.

We end the description of the model by presenting the timing of the game. At $t=1$ the principals offer contracts, defined in terms of transfers and monitoring efforts, and investment takes place. At $t=2$ income is realized, and only the agent learns it. At $t=3$, the agent pays a transfer and the principals monitor to verify and recover income.

\section{Cooperative contracting or "merged" principals}

We begin by presenting a benchmark with no strategic interaction between the principals. Thus we assume that both principals $P_{1}$ and $P_{2}$ can cooperate fully in their monitoring efforts $p_{k}$ as if they are "merged" as one, and they offer a joint contract $t(\cdot)$. For the rest of this section we will thus refer to the merged principals in the singular.

Contract setting: In this fully cooperative environment, each principal can verify the other's effort and the transfer received from the agent. Thus the merged principal chooses the monitoring efforts $p_{1}$ and $p_{2}$ to minimize the joint cost for any overall effort $q$. Remembering that $q=$ $p_{1}+p_{2}-p_{1} p_{2}$, the cost of implementing $q$ when the principals merge is denoted by $\tilde{C}(\cdot),{ }^{9}$ which

\footnotetext{
${ }^{9}$ Since the principals contract on total effort $q$, in this section and the next, we could have simply used a cost function $C(q)$ and let the principals share this total cost. As will become clear later, the main reason to introduce this function $\tilde{C}(\cdot)$ is to have a cost of effort function that is comparable across cases when principals coordinate and do not coordinate their monitoring efforts.
} 
is the solution to the cost minimization problem:

$$
\begin{aligned}
\tilde{C}(q) & =\min _{p_{1}, p_{2}} C\left(p_{1}\right)+C\left(p_{2}\right), \\
\text { s.t. } \quad q & =p_{1}+p_{2}-p_{1} p_{2} .
\end{aligned}
$$

We make the necessary technical assumptions to ensure that $\tilde{C}(\cdot)$ is well behaved and that this cost minimization is achieved for an interior symmetric solution such that $q=p(2-p)$. ${ }^{10}$ Those conditions are presented in the Appendix as Lemma 1.

The merged principal offers a joint contract based on the total monitoring effort $q$, denoted by $t(q)$, i.e., the agent is offered a menu of options from which he chooses which repayment and associated monitoring effort he prefers. ${ }^{11}$ Hence, instead of working with direct revelation mechanisms, we work here with indirect mechanisms, i.e., nonlinear schedules mapping the agent's choice of a $q$ into a payment $t$ to the principal. The Revelation Principle is then replaced by the Taxation Principle. ${ }^{12}$ The motivation for doing so is twofold. On the one hand, the theoretical work on common agency, which we will rely on later when analyzing the non-cooperative case, has stressed that direct revelation mechanisms might be of little help in competing contracts environments. Instead, the Taxation Principle still applies. ${ }^{13}$ On the other hand, direct mechanisms are artificial theoretical tools, even with a merged principal. Direct communication of the agent's type to the principal is only relevant insofar it determines the fraction $q$ of verified income and one may as well let the agent choose directly this latter payoff-relevant variable.

Recall the timing of the game. First, the principal offers the contract $t(q)$, which is a menu of ordered pairs of transfers and monitoring efforts. Second, the agent learns $\theta$. Third, given the contract and conditional on realized $\theta$, the agent chooses the pair of transfer and monitoring effort that will be implemented; monitoring takes place, the principal receives $q \theta+t(q)$, while the agent keeps the rest $\theta-q \theta-t(q)$.

Anticipating the agent's choice given a contract: We can define the agent's information

\footnotetext{
${ }^{10}$ It is worth stressing that, in the absence of such assumptions on $C(\cdot)$, cost minimization may be achieved for an asymmetric solution, for instance $p_{1}=q$ and $p_{2}=0$. This means that efficiency considerations require that only one monitor is used even when principals merge perfectly. We leave for future research the analysis of these asymmetric situations.

${ }^{11}$ Of course, these mechanisms inherit the usual caveat on commitment, and we will assume that the principal is committed to implement the $q$ that is chosen by the agent. Khalil and Parigi [18] analyze the case where the principal cannot commit to audit in a CSV model. See also Krasa and Villamil [17] who show that debt can be optimal if the principal cannot commit to enforce contracts.

${ }^{12}$ See Rochet [32].

${ }^{13}$ Martimort and Stole [25] show that focusing on the set of nonlinear prices may allow a complete characterization of pure strategy equilibria with non-random mechanisms in common agency environments similar to that we analyze here. On this see also Peters [29].
} 
rent as:

$$
U(\theta)=\max _{q}(1-q) \theta-t(q)
$$

and limited liability requires that:

$$
U(\theta) \geq 0 \text { for all } \theta
$$

The agent's private information implies that any choice $q(\theta)$ made by an agent having obtained a profit $\theta$ must be incentive compatible. The incentive compatibility constraint can be obtained from $U(\theta)$ using the Envelope Theorem; namely:

$$
\dot{U}(\theta)=1-q(\theta) \text { for all } \theta
$$

Standard revealed-preference arguments show also that $q(\cdot)$ (resp. $t(\cdot)$ ) is decreasing (resp. increasing) with respect to $\theta$ and thus a.e. differentiable with a derivative such that:

$$
\dot{q}(\theta) \leq 0 \quad \text { for all } \theta
$$

Note that (6) is also the second-order condition associated with (3). The conditions (5) and (6) capture the essence of the incentive problem and conform to familiar intuitions of contracting problems under hidden information. The only way to reduce the rent of the agent is by increasing $q(\cdot)$, and to discourage the agent from "understating" income, $q(\cdot)$ must be decreasing in $\theta$.

To see precisely how the principal influences the agent's choice of $q(\theta)$, using the a.e. differentiability of the repayment schedule $t(\cdot)$, we explicitly state the agent's first-order condition with respect to $q$ that is associated with (3):

$$
-\theta=t^{\prime}(q(\theta))<0 \quad \text { for all } \theta
$$

Therefore, to induce the agent to choose a higher $q$, the principal must reduce the repayment, by reducing the slope of $t(\cdot)$. We refer to this reduction ${ }^{14}$ in $t^{\prime}(q(\theta))$ as the marginal incentive cost of inducing a unit increase in $q(\theta)$, which is in addition to the physical marginal cost $\tilde{C}^{\prime}(q(\theta))$ borne by the principal. Exploring how this cost changes in the common agency setting will be a key theme of the paper and will help explain the various results we obtain.

Now we can present the principal's problem in the merged or cooperative benchmark, denoted thereafter by $\left(\mathcal{P}^{m}\right)$. The principal chooses the effort $q(\cdot)$ and the monetary payment $t(\cdot)$ to maximize his net payoff anticipating the agent's incentives:

$$
\begin{gathered}
\max _{\{q(\cdot), t(\cdot)\}} \int_{\underline{\theta}}^{\bar{\theta}} f(\theta)(-\tilde{C}(q(\theta))+\theta q(\theta)+t(q(\theta))) d \theta \\
\text { s.t. }(3),(4),(5) \text { and }(6) .
\end{gathered}
$$

\footnotetext{
${ }^{14}$ Since $t^{\prime}(q(\theta))$ is negative, a reduction here means making $t^{\prime}(q(\theta))$ even more negative.
} 
Using the superscript $m$ to refer to optimal values of the merged case, we present the first set of results. (See also Figure 1.)

Proposition 1 The optimal contract offered when the principals merge as one entails:

- A total monitoring effort $q^{m}(\theta)$ non-increasing in $\theta$, given by

$$
\tilde{C}^{\prime}\left(q^{m}(\theta)\right)=\frac{1-F(\theta)}{f(\theta)} \quad \text { for all } \theta
$$

with no monitoring effort applied only for the highest profit level, yielding $q^{m}(\bar{\theta})=0$.

- A total payment by the agent, $q^{m}(\theta) \theta+t^{m}(q(\theta))$, that is increasing and concave in $\theta$.

Proof In the Appendix.

Condition (8) is familiar from incentive problems with hidden information. An increase in $q(x)$ discourages "understatement" of income by all $\theta>x$. Hence, the marginal benefit of increasing $q(x)$ is the reduction of rent by a unit for all $\theta>x$, given by $1-F(x)$, which is balanced against the expected marginal cost $f(x) \tilde{C}^{\prime}(q(x))$. Monitoring is optimal except for the highest profit, and monitoring increases as outcomes get worse. Our result indicates that the agent is held to account more intensively if he pretends to have had bad luck, a feature of many instances where monitoring takes place after agents' actions.

The total payment is also concave for this very reason: at the margin, the principal's ability to increase the total payment decreases since incentive compatibility requires that $q(\cdot)$ be non-increasing. Therefore, the total payment from the agent to the merged principal resembles dividends paid to outside equity holders in that the repayment grows with profit but never absorbs all the profit. The agent is the residual claimant and his payoff resembles that of an inside equity holder that keeps an increasing proportion of profit. With better outcomes the importance of transfers to extract profit increases relative to monitoring.

If the marginal cost of monitoring were constant, say $c,(8)$ would be replaced by the condition $q(\theta)=0$ if and only if

$$
c>\frac{1-F(\theta)}{f(\theta)}
$$

and $q(\theta)=1$ otherwise. The optimal contract is then a debt contract such that the entire profit is extracted for low $\theta$ and no monitoring for high profit levels. By assuming a monitoring technology with decreasing returns we will stress in Section 5 how contracts may acquire debt-like features due to strategic reasons only. 
We define by $p^{m}(\cdot)$ the individual monitoring effort of a given principal that solves problem $P \tilde{C}$ and minimizes the total cost of monitoring given $q^{m}(\cdot)$. We have $q^{m}(\theta)=p^{m}(\theta)\left(2-p^{m}(\theta)\right)$ and it is easy to check that (8) becomes:

$$
C^{\prime}\left(p^{m}(\theta)\right)=\left(1-p^{m}(\theta)\right)\left(\frac{1-F(\theta)}{f(\theta)}\right) \text { for all } \theta
$$

where the joint cost function $\tilde{C}(\cdot)$ is replaced by the primitive individual cost function $C(\cdot)$. This expression will be useful to perform comparisons with the case where the principals do not cooperate in monitoring that we present in Section 5.

\section{Non-Cooperative Contracts and Centralized Monitoring Ef- forts}

Even if monitoring is centralized under a common governance mechanism, common agency issues can affect the outcome of the monitoring game. In this section, we illustrate this using a case where principals have direct non-cooperative financial relationships with the agent. Financiers may independently arrange for private transfers but agree to rely on a common monitoring technology to verify and recover income whose cost they bear (e.g. a bankruptcy court or a third party that monitors on their behalf). By altering one's contract, each principal affects the agent's choice and thus tries to use the agent to mislead the other principal. Therefore, even though monitoring is centralized, if the principals cannot coordinate their contracts, each principal derives a positive externality from the other principal's contract, and in equilibrium, there is more monitoring than in the cooperative case. As a result, all parties are worse off - the principals and the agent. This implies that when monitoring is centralized, it is important to limit uncoordinated direct relationships between principals and the common agent. For example, in syndicated loans the lead manager performs exactly the function of preventing side contracting between each lender and the borrower.

Contract setting: We now consider situations where the two principals choose their contracts $t_{k}(q)$ for $k=1,2$, non-cooperatively. We assume, for simplicity, that the principals share equally the cost and proceeds from centralized monitoring: each principal bears half the cost of $q, \frac{\tilde{C}(q)}{2}$, and each receives half of the recovered profit, $\frac{q \theta}{2} \cdot{ }^{15}$ As is readily seen, the analysis in this section also captures the case where the principals are able to delegate the monitoring activity (without further agency cost) and they share the cost of the delegated monitor that exerts an effort $q$.

The principals compete by simultaneously offering the nonlinear transfer schemes $t_{k}(q)$, with

\footnotetext{
${ }^{15}$ Instead of $\frac{1}{2}$, if the principals had used the fractions $\lambda$ and $(1-\lambda)$ to divide cost and extracted profit, the equilibrium level of monitoring would remain unchanged, characterized by condition (13) in Proposition 2.
} 
$k=1,2$. Given the two contracts, after observing $\theta$, the agent chooses the transfers and associated monitoring effort that will be implemented. For technical purposes, we also restrict attention to contracts which are differentiable almost everywhere. ${ }^{16}$ We will look for a Nash equilibrium in differentiable contracts $\left\{t_{1}^{c}(q) ; t_{2}^{c}(q)\right\}$ (thereafter an equilibrium) where the superscript $c$ refers to equilibrium values in the centralized monitoring case.

Externality from $P_{2}$ 's contract - anticipating the agent's choice given $t_{2}^{c}(q)$ : We intend to compute $P_{1}$ 's best response to a nonlinear contract $t_{2}^{c}(\cdot)$ offered by $P_{2}$, and the first step is to anticipate the agent's choice of $q$ to a contract offered by $P_{1}$. Rewriting the agent's problem in the case of two principals competing in transfers we obtain the following expression of the agent's information rent:

$$
U(\theta)=\max _{q}(1-q) \theta-t_{1}(q)-t_{2}^{c}(q) \quad \text { for all } \theta .
$$

Applying the Envelope Theorem to (11) again yields (5). Similarly, second-order conditions are still characterized by (6) as in the cooperative or merged principals' case of Section 3.

The strategic interaction between the principals is best illustrated by showing how $P_{1}$ 's marginal incentive cost of inducing an increase in $q$ changes compared to the case of the merged principals. For any nonlinear schedule $t_{1}(\cdot)$ offered by $P_{1}$, the agent's first-order condition associated with (11) becomes:

$$
\theta+t_{1}^{\prime}(q(\theta))+t_{2}^{c \prime}(q(\theta))=0 \text { for all } \theta
$$

Just as in the previous case, $P_{1}$ needs to lower the marginal repayment $t_{1}^{\prime}(\cdot)$ to induce a marginal increase in the choice of monitoring $q$. However, $P_{1}$ also needs now to anticipate the effect of the equilibrium contract of $P_{2}$ on the agent's behavior. Given the equilibrium contract $t_{2}^{c}(\cdot)$, an increase in $q$ at a given $\theta$ implies a movement along the schedule $t_{2}^{c}(\cdot) .{ }^{17}$ In the Appendix, we show that $t_{2}^{c}(q)$ is decreasing and convex in a symmetric equilibrium. The term $t_{2}^{c \prime}(\cdot)$ in condition (12) captures the fact that the agent pays a smaller transfer to $P_{2}$ if the agent chooses a higher monitoring intensity $q(\theta)$, given $t_{2}^{c}(\cdot)$. The intuition goes as follows: since an agent with a higher profit chooses to be monitored less $(\dot{q}(\theta) \leq 0), P_{1}$ "tricks" $P_{2}$ into believing that the realized income is lower by inducing an increase in monitoring $q(\theta)$, which induces $P_{2}$ to ask for a lower transfer.

\footnotetext{
${ }^{16}$ This is a standard restriction in common agency games. See Martimort [22] and Stole [36].

${ }^{17}$ To be more precise, any such movement along the schedule $t_{2}^{c}(\cdot)$ is only possible if this latter schedule is sufficiently extended for monitoring efforts which are out of the equilibrium set (the same will be true also in the case of decentralized monitoring efforts). These extensions are not really a difficulty here. It suffices to find a twice differentiable extension of the equilibrium schedule over the interval $[p(0), 1]$. See Martimort [22] for such an analysis.
} 
Of course, in equilibrium the principals are not tricked, but this incentive externality that each principal's contract bestows upon the other principal, leads to a greater amount of monitoring under non-cooperative contracting than in the benchmark case. If we take as a measure of total welfare the sum of the two principals and the agent's payoffs, we can easily observe that total welfare is maximized when the expected cost of the monitoring effort is minimized given that the size of profit is exogenous. ${ }^{18}$ Thus we interpret the greater amount of monitoring under non-cooperative contracting as too much monitoring.

We report the main results of this section (see also Figure 1):

Proposition 2 The unique equilibrium of the non-cooperative program where the principals coordinate in choosing their monitoring efforts is symmetric with $t_{1}^{c}(q)=t_{2}^{c}(q)=t^{c}(q)$ and it entails:

- A total monitoring effort, $q^{c}(\theta)$, given by

$$
\tilde{C}^{\prime}\left(q^{c}(\theta)\right)=2\left(\frac{1-F(\theta)}{f(\theta)}\right) \text { for all } \theta,
$$

that is non-increasing in $\theta$ with no monitoring effort for the highest profit level only, yielding $q^{c}(\bar{\theta})=0$.

- A total monitoring effort that is higher than the cooperative or merged case, $q^{c}(\theta)>q^{m}(\theta)$ for $\theta<\bar{\theta}$.

- A total payment by the agent, $\theta q(\theta)+2 t^{c}(q(\theta))$, that is increasing and concave in $\theta$; it is higher than the total payment under the merged case for all $\theta>\underline{\theta}$.

Proof In the Appendix.

Externality and excessive monitoring: Even though the incentive externality identified above plays a critical role, we need to account for two other effects to explain why there is more monitoring relative to cooperative contracting. First, monitoring is a public good as an increase in monitoring results in the other principal obtaining half of $q \theta$. Second, each principal incurs only half the marginal cost of monitoring. We show below that the reduction in $P_{1}$ 's incentive cost due to $P_{2}$ 's contract exactly negates the first effect, i.e., the loss to $P_{2}$ of half the extracted income from monitoring. Thus, it is the second effect, the shared cost of monitoring, that determines the increase in $q(\theta)$ relative to the merged principals case, and this is captured in condition (13).

\footnotetext{
${ }^{18}$ We assume that it is not possible to sell the project to the common agent. Otherwise, the best outcome would of course be to give the project to the agent and never to monitor.
} 
To see the argument more precisely, let us reproduce $P_{1}$ 's best response from the Appendix. The total effort that $P_{1}$ would like to implement in a best response to $P_{2}$ 's contract $t_{2}^{c}(\cdot)$, is given by condition (29) in the Appendix:

$$
\frac{1}{2} \tilde{C}^{\prime}(q(\theta))=\frac{1-F(\theta)}{f(\theta)}-\frac{\theta}{2}-t_{2}^{c \prime}(q(\theta)) \text { for all } \theta .
$$

It is useful to contrast this expression with condition (8) of the previous section where the principals are merged as one. In the current case, each principal pays half the marginal cost of monitoring an agent with profit $\theta$, but does not capture the entire marginal benefit $\frac{1-F(\theta)}{f(\theta)}$ from reducing the information rent of this agent in case his profit is higher than $\theta$. The other principal takes away $\frac{\theta}{2}$. The final term represents the externality that $P_{2}$ 's contract exerts on $P_{1}$ : the marginal incentive cost of inducing a unit increase in $q(\theta)$ is lowered by the amount $-t_{2}^{c \prime}(q(\theta))$ as we saw in (12). Were it not for this externality, the marginal benefit of monitoring would become negative when the agent's profit is sufficiently large, i.e., for $\theta>\theta^{*}$, where $\theta^{*}$ is defined by

$$
\frac{\theta^{*}}{2}=\frac{1-F\left(\theta^{*}\right)}{f\left(\theta^{*}\right)} .
$$

The fact that $\frac{1-F(\theta)}{f(\theta)}$ is decreasing in $\theta$ ensures that there exists a unique $\theta^{*}$ such that $\underline{\theta}<\theta^{*}<\bar{\theta}$ when $\underline{\theta} f(\underline{\theta})<2$, i.e., if $\underline{\theta}$ and/or $f(\underline{\theta})$ is small enough, an assumption that we will make from now on. The externality results in monitoring of all but the highest profit level, but without it there would be zero monitoring for profit levels greater than $\theta^{*}$.

We will argue here that the incentive externality and the "leakage" of $\frac{\theta}{2}$ to the other principal cancel each other and we obtain the identified condition (13) to characterize the outcome $q^{c}(\theta)$. Condition (12) shows that the principals share the marginal incentive cost of inducing an increase in $q$. Since the agent's utility is separable in transfers, this burden is exactly equal to the extra income extracted, where $\theta$ represents the marginal extracted income due to a unit increase in $q(\theta)$. Since the equilibrium is symmetric, we have $t^{c \prime}(q(\theta))=-\frac{\theta}{2}$. Thus $P_{1}$ 's incentive cost of inducing a marginal increase in $q$ is half the amount of what it was in the cooperative case, which was given by condition (7). Then, condition (14) shows that the marginal benefit from increasing $q(\theta)$ is exactly the same as in the cooperative case, while the marginal cost to $P_{1}$ is halved, which leads to excessive monitoring. Now we can apply the standard intuition for negative externalities: $P_{1}$ does not take into account that given the contract $t_{2}^{c}(\cdot), P_{2}$ 's return declines as $P_{1}$ induces a higher monitoring effort, which results in higher monitoring in equilibrium.

Insert Figure 1 here 
From the incentive constraint (5), we know that the rent is lower if $q(\cdot)$ is higher. Hence, common agency leads to too much extraction of the agent's information rent:

$$
U^{c}(\theta)<U^{m}(\theta) \quad \text { for all } \theta
$$

except for the lowest level of profit where those two rents are both equal to zero. All parties prefer cooperative or merged contracting to non-cooperative contracting under centralized monitoring. While total payment to each principal is higher, each principal's payoff as well as welfare (i.e., the sum of payoffs of the principals and the agent) is lower under non-cooperative contracting. Therefore, our analysis suggests that it is important to limit uncoordinated side contracts between principals and a common agent when monitoring is centralized.

Again, we can define by $p^{c}(\cdot)$ the individual monitoring effort of a given principal that solves the problem of a principal and minimizes the total cost of monitoring given $q^{c}(\cdot)$. It is easy to check that $p^{c}(\theta)$ is uniquely characterized by

$$
C^{\prime}\left(p^{c}(\theta)\right)=2\left(1-p^{c}(\theta)\right)\left(\frac{1-F(\theta)}{f(\theta)}\right) .
$$

Modeling issues: Two aspects of centralized monitoring under non-cooperative contracting encourage principals to increase monitoring. First, each principal exploits the agent's role as a communication channel to reap some benefits from the other principal's contract. Second, each principal can affect total monitoring effort $q$ directly by changing only his own contract, which relies on the assumption that each principal is committed to increase his effort as needed to support the chosen $q$. In equilibrium, this implies that each principal increases his individual effort $p_{k}$ by a unit when the other raises his by a unit, i.e., there is no free riding in monitoring efforts.

If the principals chose their individual efforts $p_{k}$ independently, the contract offered by each principal will have to be based on his own monitoring effort $p_{k}$ only instead of $q$. A principal, then, would not be able to affect $q$ directly by changing only his own contract with the agent. He would have to rely on the other principal's effort too, which would be strategically chosen and may not increase as much as desired. The positive externality from each others' contract would also be weaker. We study these issues in the next section.

\section{Non-Cooperative Contracts and Decentralized Monitoring Ef- forts}

In a monitoring context where no common institution can be constructed to extract the returns of the project, the theme of free riding in monitoring efforts becomes central. This issue could not be addressed under centralized monitoring since we assumed that the principals could verify each 
other's efforts. Here we allow the principals to choose their efforts independently. For example each principal could hire his own lawyers to put pressure on the agent to pay out income even if the total amount of income that can be extracted is the result of the joint efforts. Hence, we consider a situation with an even greater incompleteness of contracts than in the previous section: not only do the principals not coordinate in choosing transfers, but each principal does not observe the effort of the other in monitoring the common agent. We find that there will be free riding in monitoring efforts, which means that a unit increase in $p_{k}$ is associated with a less than one unit increase in $p_{j \neq k}$. This leads to a number of results. First, there is less monitoring than in the case of centralized monitoring which makes the agent better off. Second, and perhaps more striking is the existence of equilibria where the monitoring level is lower than under the merged principals benchmark. That is non-cooperative contracting does not necessarily lead to too much monitoring. Third, free riding in monitoring efforts leads to a collapse of monitoring for high profit levels; there is no monitoring if profit is above a threshold and principals rely instead on a constant transfer to extract income in these states. This happens mainly because free riding in monitoring weakens the incentive externality between the principals.

Contract setting: Under this setting, which we refer to as decentralized monitoring, the principals' monitoring efforts $p_{k}$ for $k=1,2$ are set non-cooperatively, and one principal's effort $p_{k}$ is unobservable by the other principal. Each principal incurs his own cost of effort $C\left(p_{k}\right)$. Hence the indirect mechanisms we consider stipulate a repayment, denoted by $t_{k}\left(p_{k}\right)$, to each $P_{k}$ based directly on his monitoring effort. Given the pair of contracts $t_{k}\left(p_{k}\right)$ for $k=1,2$, the agent picks the transfer and associated monitoring effort to be implemented by each principal and privately discloses the pair $\left(t_{k}, p_{k}\right)$ only to $P_{k}$. The principals share equally the fraction $q$ of the extracted profit $\theta$. We will look for a Nash equilibrium in differentiable contracts $\left\{t_{1}^{d}\left(p_{1}\right), t_{2}^{d}\left(p_{2}\right)\right\}$ where the superscript refers to the equilibrium values for the decentralized case.

Free riding in monitoring — anticipating the agent's choice given $t_{2}^{d}\left(p_{2}\right)$ : Again, we want to compute the best response of $P_{1}$ given the equilibrium offer $t_{2}^{d}(\cdot)$ of principal 2 . A key step is to anticipate the agent's choice of $p_{2}$ for any given $p_{1}$ that $P_{1}$ wants to implement. For this, it is useful to first define the following indirect utility function, given $P_{2}$ 's equilibrium contract:

$$
v\left(p_{1}, \theta\right)=\max _{p_{2}}\left(1-p_{1}\right)\left(1-p_{2}\right) \theta-t_{2}^{d}\left(p_{2}\right) \quad \text { for all }\left(p_{1}, \theta\right) .
$$

Note that $1-q=\left(1-p_{1}\right)\left(1-p_{2}\right)$, remembering the definition of $q$. The function $v(\cdot)$ represents the agent's earning aside from the transfer payment to principal $P_{1}$, given $P_{2}$ 's contract $t_{2}^{d}(\cdot)$. This is all that matters from $P_{1}$ 's point of view to assess the costs and benefits of changing his own contract with the agent. Note that treating $v(\cdot)$ as the agent's privately known "income", we can consider $P_{1}$ 's problem with the agent as a "one principal-one agent" problem. We first characterize the agent's choice of $p_{2}$, denoted by $p_{2}^{*}\left(p_{1}, \theta\right)$, for any $p_{1}$ that $P_{1}$ could want to implement, and 
then we present $P_{1}$ 's problem in which this response $p_{2}^{*}\left(p_{1}, \theta\right)$ is anticipated.

The maximand in (17) is concave if $t_{2}^{d \prime \prime}(\cdot)$ is positive, and in the Appendix we show this to be true in equilibrium. ${ }^{19}$ Assuming this for now, we obtain the first-order condition with respect to $p_{2}$ that defines the agent's choice $p_{2}^{*}\left(p_{1}, \theta\right)$ when it is positive:

$$
\theta\left(1-p_{1}\right)=-t_{2}^{d \prime}\left(p_{2}^{*}\left(p_{1}, \theta\right)\right) \text { for all }\left(p_{1}, \theta\right) \text {. }
$$

Note that we can also use this condition to calculate the marginal incentive cost of inducing an increase in monitoring effort just like we did in the previous sections. Again, the reduction in $t_{2}^{d}(\cdot)$ captures $P_{2}$ 's marginal incentive cost of inducing a unit increase in $p_{2}$ on top of the direct marginal cost $C^{\prime}\left(p_{2}\right)$. Condition (18) shows that this incentive cost for $P_{2}$ is inversely related to the other principal's effort $p_{1}$. This is because of wasteful duplication in principals' efforts, $\frac{\partial^{2} q}{\partial p_{1} \partial p_{2}}<0$ : in response to an increase in $p_{1}$, the agent increases $p_{2}$ to reduce $q$ the part of income that will be verified. ${ }^{20}$ In the Appendix, we also show that $\frac{\partial p_{2}^{*}}{\partial p_{1}}<1$ in equilibrium, which is rather intuitive. It simply means that although $p_{1}$ and $p_{2}$ are complements, they are only imperfect complements. In the centralized case of Section 4, we assumed that each principal could affect the total monitoring effort $q(\cdot)$ by his choice of $t_{k}(\cdot)$, given the other principal's contract. Thus, each principal's monitoring effort was identically affected and in that case $\frac{\partial p_{2}^{*}}{\partial p_{1}}$ equaled one by assumption. In contrast, under decentralized efforts, principals cannot verify each other's effort, and thus each can affect only his own individual effort $p_{k}(\cdot)$. Then, we find that each principal only increases his own $p_{k}(\cdot)$ by less than a unit for a unit increase in the other principal's effort $p_{j}(\cdot)$. This means that the principals free ride on each other when providing monitoring efforts. As we will see below, it implies that the incentive externality from the other principal's contract is weakened, which in turn leads to low levels of monitoring and high profit levels not being monitored at all.

The result that the principals' efforts are imperfect complements is implied by the fact that the principals cannot verify each other's efforts, and they free ride in providing incentive to the agent to choose higher monitoring efforts. To see why, let us return to the centralized monitoring case where the principals can verify each others efforts. Instead of viewing the transfers to each principal as functions of the overall effort level $q$, one may as well (using the fact that principals can verify each others' efforts) view these transfers as functions of the individual effort levels,

\footnotetext{
${ }^{19}$ As we will see below, the equilibrium schedule $t_{2}^{d}(\cdot)$ is only right differentiable at zero and a more precise analysis is called for to take into account this corner issue. We address this issue in the Appendix.

${ }^{20}$ If instead of assuming wasteful duplication in monitoring effort, we had assumed that $q=p_{1}+p_{2}+p_{1} p_{2}$, we would have $\frac{\partial p_{2}^{*}}{\partial p_{1}}<0$, i.e., monitoring efforts would be strategic substitutes. Free riding would then take the extreme form of crowding out the other principal's effort. In equilibrium, there would be even less monitoring and a larger interval without monitoring. However, ensuring that the agent's problem is globally concave is more difficult with strategic substitutes than with strategic complements. See Martimort [22] and Stole [36] for more on this topic.
} 
which by symmetry is the same for both principals, namely $p$ such that $q=(2-p) p$. Then, the contracts $t^{*}(q)=t^{c}((2-p) p)$ form an equilibrium pair. The agent's choice of a common probability of monitoring $p(\theta)$ now solves

$$
p(\theta)=\arg \max _{p}(1-p)^{2} \theta-2 t^{*}(p) \text { for all } \theta,
$$

which leads to the first order condition

$$
\theta\left(1-p^{*}(\theta)\right)=-t^{* \prime}\left(p^{*}(\theta)\right) \quad \text { for all } \theta
$$

which is just (12) rewritten as a symmetric equilibrium. Let us now return to the decentralized case where principals cannot verify each others efforts. If one principal, say $P_{2}$, were to offer $t^{*}\left(p_{2}\right)$, the other principal will deviate from $t^{*}\left(p_{1}\right)$ and induce the agent to choose $p_{2}^{*}\left(p_{1}, \theta\right)$ such that $\frac{\partial p_{2}^{*}}{\partial p_{1}}=\frac{\theta}{t^{* \prime \prime}\left(p_{2}^{*}\left(p_{1}, \theta\right)\right)}<1$. This is derived by using $t^{*}(\cdot)$ in (18) and noting that by differentiating (19) with respect to $\theta$ implies $t^{* \prime \prime}\left(p^{*}(\theta)\right)=\frac{-1+p^{*}(\theta)+\theta \dot{p}^{*}(\theta)}{\dot{p}^{*}(\theta)}$.

Now we move on to set up and solve $P_{1}$ 's problem. Standard methods for a single-principal problem can be employed remembering that, given $t_{2}^{d}(\cdot)$, the agent's retained earning equals $v\left(\theta, p_{1}\right)$. Using the indirect utility formulation in (17), the agent's rent is rewritten as

$$
U(\theta)=\max _{p_{1}} v\left(p_{1}, \theta\right)-t_{1}\left(p_{1}\right) \quad \text { for all } \theta
$$

from which we obtain by the Envelope Theorem

$$
\begin{aligned}
\dot{U}(\theta) & =v_{\theta}\left(p_{1}(\theta), \theta\right) \\
& =\left(1-p_{1}(\theta)\right)\left(1-p_{2}^{*}\left(p_{1}(\theta), \theta\right)\right) \text { for all } \theta
\end{aligned}
$$

where $v_{\theta}(\cdot)$ denotes the partial derivative of $v(\cdot)$ with respect to $\theta$. Even though this incentive constraint has a form similar to that of the previous incentive constraint (5), it now depends on $p_{2}^{*}\left(p_{1}(\theta), \theta\right)$ which in turn depends on the endogenously determined schedule $t_{2}^{d}(\cdot)$. Hence, the local second-order sufficient condition $(S O S C)$ may appear to be more complex than in the centralized case. However, since $p_{1}$ and $p_{2}$ are complements, we show in the Appendix that the local SOSC can be simplified to get the simple monotonicity condition:

$$
\dot{p}_{1}(\theta) \leq 0 \text { for all } \theta \text {. }
$$

Note that the corresponding total effort $q$ in a symmetric equilibrium where both principals exert the same effort can be written as $q^{d}(\theta)=p^{d}(\theta)\left(2-p^{d}(\theta)\right)$. Using this notation we can state the following results that characterize our main findings regarding decentralized monitoring. 
Proposition 3 When the principals compete in both contracts and monitoring efforts, there is a continuum of symmetric equilibria assuming concavity of each principal's problems. ${ }^{21}$ Every symmetric equilibrium entails:

- A monitoring effort $p^{d}(\theta)$ for each principal which is non-increasing in $\theta$; there exists $\hat{\theta}$ such that $\underline{\theta}<\hat{\theta}<\bar{\theta}$ and $p^{d}(\theta)=0$ for $\theta>\hat{\theta}$, i.e., no monitoring occurs for profit levels above $\hat{\theta}$.

- A total monitoring effort that is smaller than in the centralized case, $q^{d}(\theta)<q^{c}(\theta)$ for $\theta \in(\underline{\theta}, \bar{\theta}) .^{22}$

- A total payment, $\frac{q \theta}{2}+t_{k}\left(p_{k}(\theta)\right)$, from the agent to each principal that is non-decreasing and concave in $\theta$ with payments being constant for $\theta>\hat{\theta}$; this total payment is smaller than the payment in the centralized case for all $\theta>\underline{\theta}$.

Proof In the Appendix.

Free riding and the outcome - sketch of the arguments: It is precisely the free riding in monitoring efforts, $\frac{\partial p_{2}^{*}}{\partial p_{1}}<1$, that generates the difference in results between the centralized and decentralized cases. Free riding has an impact via two sources: (i) a unit increase in $p_{1}$ results in less than a unit increase in $q$, and (ii) the incentive externality, which is the reduction in the cost of providing incentive due to the other principal's contract, is also smaller. The first effect results in a lower amount of monitoring relative to the centralized case, and the second results in the collapse of monitoring for high income levels.

We can provide some details of the argument by focusing on the condition determining the optimal $p_{1}$ that $P_{1}$ would like to implement as a best response to $t_{2}^{d}(\cdot)$, the equilibrium contract of $P_{2}$. This condition is given as (32) in the Appendix. Suppressing arguments of functions where obvious, we can rearrange condition (32) into the following condition:

$$
C^{\prime}\left(p_{1}^{d}\right) \geq\left[\left(1-p_{2}^{*}\right)+\frac{\partial p_{2}^{*}}{\partial p_{1}}\left(1-p_{1}^{d}\right)\right]\left(\frac{1-F}{f}-\frac{\theta}{2}\right)-\frac{\partial p_{2}^{*}}{\partial p_{1}} t_{2}^{d \prime}\left(p_{2}^{*}\right)
$$

where the right hand side represents the marginal benefit of increasing $p_{1} \cdot{ }^{23}$ This marginal benefit is composed of two expressions, with the first one reflecting the impact on $q$ and the extraction of income, while the second captures the effect of the externality from $P_{2}$ 's contract. Now we examine each of these two parts in detail starting with the first. Note that $\left(1-p_{2}\right)$ is the derivative of $q$

\footnotetext{
${ }^{21}$ The exact condition is (35) in the appendix.

${ }^{22}$ The equilibrium schedule $q^{d}(\theta)$ that begins at $q^{c}(\underline{\theta})$ has infinite slope at $\underline{\theta}$; for all other solutions $q^{d}(\theta)$, we have $q^{d}(\theta)<q^{c}(\theta)$ for $\theta<\bar{\theta}$. Finally, $q^{d}(\bar{\theta})=q^{c}(\bar{\theta})=0$ for every solution $q^{d}(\theta)$.

${ }^{23}$ The inequality in (23) is used to capture the fact that, in this decentralized contracting setting, the optimal solution can be at a zero corner.
} 
with respect to $p_{1}$. An increase in $p_{1}$ increases $q$ directly, and also indirectly via $p_{2}^{*}(\cdot)$; the terms in the square brackets capture this increase in $q$. Note that the effect on $q$ via $p_{2}^{*}(\cdot)$ is a bit muted due to free riding. As a result of an increase in $q$, the agent's rent is reduced by $\frac{1-F}{f}$, but $\frac{\theta}{2}$ goes to $P_{2}$. The term $-\frac{\partial p_{2}^{*}}{\partial p_{1}}(\cdot) t_{2}^{d \prime}(\cdot)>0$ represents the positive incentive externality from $P_{2}$ 's contract. This externality is different from that under centralized monitoring since now $P_{2}$ can only observe a change in his own effort $p_{2}$, whereas under centralized monitoring it was assumed that he could observe changes in total effort $q$. An increase in $p_{1}$ leads to an increase in $p_{2}$, and given his equilibrium contract $t_{2}^{c}(\cdot), P_{2}$ asks for a lower transfer. But because $\frac{\partial p_{2}^{*}}{\partial p_{1}}<1$, the positive externality stemming from the other principal's contract is somewhat counteracted by a negative externality related to this free riding phenomenon. Without the positive externality $\left(-\frac{\partial p_{2}^{*}}{\partial p_{1}}(\cdot) t_{2}^{d \prime}(\cdot)\right)$, monitoring would end at $\theta^{*}$, defined by condition (15), due to the leakage of $\frac{\theta}{2}$.

To see the effect of $\frac{\partial p_{2}^{*}}{\partial p_{1}}<1$ more clearly, let us substitute $t_{2}^{d \prime}(\cdot)$ in (23) using (18), and note that in a symmetric equilibrium we have $p_{1}^{d}(\theta)=p_{2}^{*}\left(p_{1}(\theta), \theta\right)$. We can then rewrite (23) as:

$$
C^{\prime}\left(p_{1}^{d}\right) \geq\left(1-p_{1}^{d}\right)\left(\frac{1-F}{f}\left(1+\frac{\partial p_{2}^{*}}{\partial p_{1}}\right)+\frac{\theta}{2}\left(\frac{\partial p_{2}^{*}}{\partial p_{1}}-1\right)\right)
$$

Condition (24) precisely captures the difference between the centralized and the decentralized cases of monitoring. Indeed, under centralized monitoring, the minimization of the monitoring costs forces principals to credibly commit to offer the same monitoring effort and thus $\frac{\partial p_{2}^{*}}{\partial p_{1}}=1$. Inserting $\frac{\partial p_{2}^{*}}{\partial p_{1}}=1$ in condition (24) yields $p^{d}(\theta)=p^{c}(\theta)$, as can be verified by remembering (16). Of course, the non-observability of the monitoring effort of $P_{2}$ by $P_{1}$ makes such commitment impossible; in equilibrium, we have free riding and $\frac{\partial p_{2}^{*}}{\partial p_{1}}<1$. The difference between $p^{d}(\theta)$ and $p^{c}(\theta)$ reflects this new incompleteness. Since $p_{2}$ and $p_{1}$ are only imperfect complements, one would suspect that there will be less monitoring in the decentralized case, i.e., $p^{d}(\theta)$ will lie below $p^{c}(\theta)$.

While the above comparison is quite intuitive, it is not all that can be inferred from inserting $\frac{\partial p_{2}^{*}}{\partial p_{1}}<1$ in condition (24). Note that the marginal benefit of raising $p_{1}$ is negative for $\theta$ close to $\bar{\theta}$, and hence, there is no monitoring for high values of $\theta$. Then condition $(21)$ implies that $\dot{U}^{d}(\theta)=1$ for these values, and we immediately obtain that the total payment is constant for such levels of profits. Under centralized monitoring, we found that the incentive externality from the other principal was large enough in condition (14) to make monitoring optimal for all $\theta<\bar{\theta}$. Here, with $\frac{\partial p_{2}^{*}}{\partial p_{1}}<1$, the incentive externality is not strong enough to overcome the leakage of $\frac{\theta}{2}$ to the other principal for all income levels. Since $p_{k}$ decreases with $\theta$, condition (18) shows that the marginal incentive cost increases with $\theta$, and each principal finds it prohibitively costly to implement a positive level of monitoring for high $\theta$.

Insert Figure 2 here 
With centralized monitoring, the principals are forced to compete only in the repayments they request from the common agent. A deviation by either principal from the cooperative outcome can only take the form of a marginal decrease in the repayment asked by this principal in exchange of a higher monitoring effort. Since principals only bear half of the joint cost of monitoring, they tend to monitor excessively. Instead, with decentralized monitoring, the principals compete both in the repayments they request from the agent and the monitoring effort. Since each principal benefits from an increase in total monitoring effort, but only contributes partially to it, there is a free-riding problem and individual monitoring efforts decrease with respect to the centralized setting. In particular, this free riding makes monitoring prohibitively costly when income is high and principals rely on constant transfers.

Structure of equilibria: Having said that the upper bound on the set of outcomes $p^{d}(\theta)$ is given by the hypothetical case, $\frac{\partial p_{2}^{*}}{\partial p_{1}}=1$, we now argue that the lower bound is given by the hypothetical opposite, $\frac{\partial p_{2}^{*}}{\partial p_{1}}=0$. In this case the common agent would not change the amount of monitoring he chooses for $P_{2}$ as $P_{1}$ changes his own effort $p_{1}$, i.e., $\frac{\partial p_{2}^{*}}{\partial p_{1}}=0$. This contractual outcome can be viewed as an "equilibrium" of a quasi-game where each principal is "myopic" and believes (wrongly) that he cannot influence the agent's choice within the menu proposed by the other principal. In that case, the "equilibrium" would be obtained by inserting $\frac{\partial p_{2}^{*}}{\partial p_{1}}=0$ in $(24)$, and it would entail a symmetric monitoring effort $p_{L}(\theta)$ defined by

$$
C^{\prime}\left(p_{L}(\theta)\right)=\left(1-p_{L}(\theta)\right)\left(\frac{1-F}{f}-\frac{\theta}{2}\right)
$$

for $\theta \leq \theta^{*}$ and zero otherwise, where $\theta^{*}$ is defined by (15). Of course, the common agent always modifies his choice of $p_{2}$ (even if it is only by a small amount in some equilibria corresponding to low levels of monitoring) when $P_{1}$ changes his own $p_{1}$. Thus one suspects that any equilibrium outcome $p^{d}(\theta)$ always lies above $p_{L}(\theta)$.

In the Appendix, we show that these intuitions are indeed correct, and that all symmetric equilibria can be ranked with respect to the monitoring effort. Any such equilibrium lies within these two boundaries $p_{L}(\theta)$ and $p^{c}(\theta)$ defined above. Note that, as principals behave more "myopically", contracts look more like debt contracts with larger flat parts.

The fact that there exists a continuum of equilibria is indicative of the prominent role that players' expectations play in this decentralized environment. In the low equilibria, close to $p_{L}(\theta)$, each principal monitors little because he expects the other to also do the same, so that $\frac{\partial p_{2}^{*}}{\partial p_{1}}$ is close to zero and his own best response is close to $p_{L}(\theta)$. Alternatively, the non-linear equilibrium schedule $t^{d}(\cdot)$ is extremely convex for such equilibria $\left(t^{d \prime \prime}(\cdot)\right.$ is high) so that principals almost commit not to change their monitoring efforts too much as $\theta$ varies.

Instead, for high equilibria, close to $p^{c}(\theta)$, each principal monitors a lot because he expects 
the other to do so as well, and his own best response is close to $p^{c}(\theta)$. The non-linear equilibrium schedule $t^{d}(\cdot)$ is less convex $\left(t^{d \prime \prime}(\cdot)\right.$ is small) so that the agent reacts a lot to a change in monitoring effort by each principal. If a principal, say $P_{1}$, envisions deviating by reducing his individual monitoring effort, the agent will choose to reduce also significantly the monitoring effort he requests of $P_{2}$. We are close to the case of centralized monitoring, which is characterized by an over-provision of monitoring effort.

Since there is less monitoring, less information rent is extracted under decentralized monitoring, compared to the centralized case, and we have:

$$
U^{c}(\theta)<U^{d}(\theta) \text { for all } \theta>\underline{\theta}
$$

Being forced to compete also in monitoring efforts to extract income makes the principals weaker: as the proverb goes "the onlooker gets the better of a fight", and the agent is better off.

The equilibrium under decentralized monitoring can be also compared with the cooperative outcome achieved under the merged principals benchmark. In particular we obtain the following result:

Proposition 4 Assume that $\frac{d}{d \theta}\left(\frac{1-F(\theta)}{f(\theta)}\right) \geq-\frac{1}{2}$. Noting that $p_{L}(\theta)$ is defined by $(25)$, take any symmetric equilibrium $p^{d}(\theta)$ under decentralized monitoring:

- if $p^{d}(\underline{\theta}) \in\left[p_{L}(\underline{\theta}), p^{m}(\underline{\theta})\right]$, then $p^{d}(\theta)<p^{m}(\theta)$ for all $\theta$ in $[\underline{\theta}, \bar{\theta}]$ (there exist decentralized equilibria where there is less monitoring than under the cooperative or merged principals case).

- if $p^{d}(\underline{\theta}) \in\left(p^{m}(\underline{\theta}), p^{c}(\theta)\right)$, then there exists $\tilde{\theta}$ such that $\underline{\theta}<\tilde{\theta}<\bar{\theta}$ and $p^{d}(\theta) \geq p^{m}(\theta)$ if and only if $\theta \in[\underline{\theta}, \tilde{\theta}]$ (there is no symmetric decentralized equilibrium that involves more monitoring than under the merged principals case for all profit levels).

Proof In the Appendix.

The proposition shows that equilibria under decentralized contracting exhibit a strong tendency towards low level of monitoring. As long as the hazard rate does not decline too quickly, $\frac{d}{d \theta}\left(\frac{1-F(\theta)}{f(\theta)}\right) \geq-\frac{1}{2}$, the decentralized equilibria $p^{d}(\theta)$ that start below $p^{m}(\theta)$, stay below $p^{m}(\theta)$ throughout. Even those equilibria corresponding to relatively flat nonlinear schedules $t(q)$ (i.e., those with a high $p^{d}(\underline{\theta})$ ) fall below the cooperative outcome for sufficiently high levels of profit. This feature of the equilibrium captures again the two externalities at play when principals do not merge. On the one hand, there might be excessive monitoring because each principal only bears half of the incentive cost from monitoring exactly as if the monitoring effort was verifiable by both 
principals. This effect might dominate for low levels of profit, i.e., when monitoring is crucial at least for some equilibria. On the other hand, there is too little monitoring because of free riding when monitoring efforts are non-verifiable and this effect always dominates in any equilibrium for sufficiently high levels of profits, i.e., when monitoring is less of an issue anyway.

Welfare: Recall that if we take as measure of total welfare the sum of the two principals and the agent's payoffs, we can easily observe that total welfare is maximized when the expected cost of the monitoring efforts is minimized. The ranking of equilibria that we have found above leads us immediately to state that welfare is maximum in the lowest symmetric equilibrium of the decentralized case. In particular it is larger than even that under the merged case since some symmetric equilibria lie strictly below $p^{m}(\theta)$ for all $\theta$ in $[\underline{\theta}, \bar{\theta})$ (i.e., those such that $p^{d}(\underline{\theta}) \in\left[p_{L}(\underline{\theta}), p^{m}(\underline{\theta})\right]$ under the assumption of Proposition 4). Also, every symmetric decentralized equilibrium dominates the equilibrium under centralized monitoring from a welfare point of view. Thus, in our framework, if contracting cannot be coordinated, welfare is higher when monitoring is also uncoordinated. While the welfare effect of a merger between two principals with centralized monitoring is positive, a merger of two principals that compete both in contracts and monitoring efforts has ambiguous welfare implications.

Profit of the principals: It is also interesting to compare the different cases or institutional arrangements in terms of the aggregate profits of the principals. Given that the principals are indispensable providers of the initial up-front investment, this may be viewed as a more relevant measure of welfare. Since $\theta$ is exogenous, the best arrangement should achieve the minimal value of the overall costs, including first monitoring costs but also the expected information rent left to the agent. The best arrangement would be our benchmark where the principals merge and minimize the sum of these two costs at $p^{m}(\theta)$. Note that even though some decentralized outcomes $p^{d}(\theta)$ have lower amount of monitoring than $p^{m}(\theta)$ (Proposition 4), these decentralized outcomes are inferior to $p^{m}(\theta)$ from the principals' viewpoint as they do not adequately account for the cost of rent left to the agent.

The comparison between decentralized and centralized monitoring is less trivial and an interesting trade-off emerges. Indeed, we know from Proposition 4 that, in some equilibria $p^{d}(\theta)$ is closer to the cooperative outcome $p^{m}(\theta)$ than $p^{c}(\theta)$, for low levels of profits whereas $p^{c}(\theta)$ is closer to $p^{m}(\theta)$ for high levels close to $\bar{\theta}$. This suggests that centralized (resp. decentralized) monitoring may be preferred when the distribution of profit puts enough (resp. little) weight on high levels of profits. Using this intuition, we construct in the Appendix (subsection 7.6) an example to show that there exist parameter configurations such that the sum of profits for the two principals can be higher under either centralized or decentralized monitoring. Therefore, either monitoring arrangement could be preferable from the principals' viewpoint depending on the underlying 
fundamentals of the problem.

\section{Conclusion}

We have considered the problem of multiple principals attempting to obtain income from a privately informed agent and designing their respective financial contracts non-cooperatively. A contribution of our analysis is to note that information problems between the monitors can affect financial contracts. The degree of coordination between these principals, stemming from the observability of their monitoring efforts, has strong implications for the shapes of financial contracts. Equity is more useful when there are few information problems between principals and monitoring can be credibly delegated or centralized. This is more likely to happen in publicly-traded companies where there are rules to guarantee a coordinated access to profits. When instead the idiosyncratic nature of the activity financed (e.g. in privately held companies) makes it impossible to rely on a common set of monitoring rules and forces the principals to monitor independently, free riding will render monitoring so costly to make it convenient to use debt-like payments where high levels of income are not monitored at all. Our finding is consistent with a well known result from the corporate finance literature (see for example the survey by Harris and Raviv [14]) according to which debt is more likely used than equity in situations with information problems. This is because debt is relatively insensitive to firm performance and is likely to be priced more accurately than equity in situations involving asymmetric information.

Our analysis could be extended along several lines. First, we have abstracted from any moral hazard issues by assuming that the size of profit is exogenous and not affected by any effort from the agent. Those considerations would lead to more complex modelling issues where principals have a common interest in inducing the agent to exert effort. The common agency equilibrium would highlight not only the features described here but also would exhibit some free riding between the principals in providing the agent correct incentives to exert effort as in pure common agency environments with moral hazard. ${ }^{24}$ One may guess that this added scope for free riding will reduce the agent's effort and thus his profit, making monitoring by the principals less of an issue.

Second, a natural extension of our framework should account for some asymmetry between the principals who could differ in terms of their cost of monitoring or according to the dates at which they contract with the agent. Asymmetry could be useful in explaining how the degree of monitoring performed by different principals is linked to their priority in contracting with the agent.

\footnotetext{
${ }^{24}$ See Bernheim and Whinston [2].
} 
Third, while our approach is similar to the CSV literature, there are two differences. In the CSV literature a strong emphasis is put on direct revelation mechanisms: the privately informed agent makes a report of his privately realized income and an audit is used to ex post check the veracity of this claim through a costly bankruptcy procedure. In our model instead, we focus on indirect mechanisms, or menus linking the repayments made to the monitoring efforts. As mentioned above although the two approaches are equivalent in the case of a single principal, the use of direct revelation mechanisms is restrictive in common agency environments as shown by Peters ([29], [30]) and Martimort and Stole [24]. The second difference with the CSV approach is that our model of monitoring is not limited to an interpretation of auditing and bankruptcies alone. Instead we allow for a broader interpretation by defining monitoring as a range of actions that occur ex post to verify and recover realized income, an approach that can be applied to model ongoing monitoring activity in healthy enterprises. ${ }^{25}$ However, it is possible to relate our first two models directly to the CSV approach and the outcomes would be identical. We would only need to specify the contract differently. If we interpret $q$ as the probability of an audit that reveals true income, then we can specify a repayment function $r(q)$ when there is no audit. Then our repayment function $t(q)=(1-q) r(q)$ is interpreted as the payment unconditional on an audit. ${ }^{26}$

Fourth, we could also extend the number of principals involved. As this number increases, the various effects stressed in the paper are exacerbated. In the case of centralized monitoring, the intensity of monitoring increases quickly with this number, making repayment a less useful screening tool when principals are dispersed but remain strategic. As far as decentralized contracting is concerned, free riding is more of a problem as the number of principals increases. One might expect that principals will behave more myopically in such environments and that the no-monitoring regions would be enhanced.

Finally, although our focus has been on financial contracting, this framework can be used to analyze other instances in which several principals monitor a common agent, like in labor contexts, banking supervision, consumer credit, development aid agencies, tax authorities, etc. Since tax collection also involves extracting income from an informed agent, our model can also be used to analyze the issues of competition between tax jurisdictions. Our analysis shows that tax collection by different jurisdictions depend on their ability to coordinate both the tax schedules and their collection efforts, an issue often neglected in the discussions over the harmonization of taxation across countries. More generally ours is an example of an agency problem with multiple con-

\footnotetext{
${ }^{25}$ See Hart [15] for a critique of the CSV approach. He also observes that the audit costs implied by the CSV approach may be too large to be consistent with observed bankruptcy costs.

${ }^{26}$ Our model of decentralized monitoring is not amenable to an interpretation of a CSV model because the $q$ depends on both principals' efforts $p_{1}$ and $p_{2}$, so that $r(\cdot)$ would become a function of the unverified effort of the other principal.
} 
stituencies. The strategic interactions between them shape the governance arrangements between the various stakeholders of a firm as well as some of the institutional mechanisms that society develops to monitor individuals.

\section{Appendix}

\subsection{Proof of Lemma 1}

Lemma 1 If $C^{\prime \prime}\left(p_{k}\right)>\frac{C^{\prime}\left(p_{k}\right)}{\left(1-p_{k}\right)}$, then there exists a unique solution to the problem $(P \tilde{C})$, with $\tilde{C}(q)=2 C(1-\sqrt{1-q})$, and $\tilde{C}(q)$ strictly convex with $\tilde{C}^{\prime}(0)=0$, and $\tilde{C}^{\prime \prime}(0)>0$.

\section{Proof}

Use the constraint in problem $P \tilde{C}$ to replace $p_{2}$ in the maximand

$$
\min _{p_{1}} C\left(p_{1}\right)+C\left(\frac{q-p_{1}}{1-p_{1}}\right)
$$

Then differentiating w.r.t. $p_{1}$ yields the FOC (after replacing $p_{2}$ )

$$
\left(1-p_{1}\right) C^{\prime}\left(p_{1}\right)=\left(1-p_{2}\right) C^{\prime}\left(p_{2}\right)
$$

If $C^{\prime \prime}\left(p_{k}\right)>\frac{C^{\prime}\left(p_{k}\right)}{\left(1-p_{k}\right)}$, the expression $\left(1-p_{k}\right) C^{\prime}\left(p_{k}\right)$ is increasing in $p_{k}$ and the maximand is quasiconvex. Therefore we have a unique symmetric solution where $p_{1}^{*}=p_{2}^{*}=1-\sqrt{1-q}$ and $\tilde{C}(q)=$ $2 C(1-\sqrt{1-q})$. Also, note that $\tilde{C}(\cdot)$ is convex

$$
\tilde{C}^{\prime}(q)=\frac{1}{\sqrt{1-q}} C^{\prime}(1-\sqrt{1-q})
$$

and

$$
\tilde{C}^{\prime \prime}(q)=\frac{C^{\prime}(1-\sqrt{1-q})}{2(1-q)^{\frac{3}{2}}}+\frac{C^{\prime \prime}(1-\sqrt{1-q})}{2(1-q)}>0,
$$

with $\tilde{C}^{\prime}(0)=2 C^{\prime}(0)=0$, and $\tilde{C}^{\prime \prime}(0)=2 C^{\prime \prime}(0)>0$.

\subsection{Proof of Proposition 1}

We reproduce the principal's problem:

$$
\begin{gathered}
\max _{\{q(\cdot), t(\cdot)\}} \int_{\underline{\theta}}^{\bar{\theta}} f(\theta)(-\tilde{C}(q(\theta))+\theta q(\theta)+t(q(\theta))) d \theta \\
\text { s.t. }(3),(4),(5) \text { and }(6) .
\end{gathered}
$$


As it is standard in the principal-agent literature, we will first neglect the constraint (6) and check later that it is satisfied by our solution. Using the incentive constraint (5), direct integration yields

$$
U(\theta)=U(\underline{\theta})+\int_{\underline{\theta}}^{\theta}(1-q(x)) d x
$$

and thus, taking expectations,

$$
\int_{\underline{\theta}}^{\bar{\theta}} U(\theta) f(\theta) d \theta=U(\underline{\theta})+\int_{\underline{\theta}}^{\bar{\theta}}(1-q(\theta))(1-F(\theta) d \theta,
$$

where the final equality is derived using integration by parts.

Standard arguments show that the principal's payoff is maximized when $U(\underline{\theta})=0$. Using (3) to replace $t(\cdot)$ in the principal's objective function and using (26), we can finally simplify the principal's problem $\left(\mathcal{P}^{m}\right)$ to obtain:

$$
\max _{q(\cdot)} \int_{\underline{\theta}}^{\bar{\theta}}\left(-\tilde{C}(q(\theta))+\theta-(1-q(\theta))\left(\frac{1-F(\theta)}{f(\theta)}\right)\right) f(\theta) d \theta .
$$

By Lemma 1, we have $\tilde{C}(\cdot)$ convex so that the principal's problem is concave. Point-wise optimization of this maximand yields the first-order condition (8) :

$$
\tilde{C}^{\prime}\left(q^{m}(\theta)\right)=\frac{1-F(\theta)}{f(\theta)}
$$

giving the optimal monitoring effort for each $\theta$.

Note that our Inada assumptions on the cost function implies that $q$ is positive for all types except the highest profit one $\bar{\theta}$, and $0<q<1$ for all other types. Since $\frac{1-F(\theta)}{f(\theta)}$ is non-increasing and $\tilde{C}^{\prime \prime}(q)>0$, the monotonicity condition (6) is satisfied by $q^{m}(\theta)$.

The total payment from the agent to the principal is $q^{m}(\theta) \theta+t^{m}(q(\theta))$, and we show below that it is increasing and concave in $\theta$. Since the agent's information rent for each $\theta$ is:

$$
U^{m}(\theta)=\int_{\underline{\theta}}^{\bar{\theta}}\left(1-q^{m}(x)\right) d x
$$

from (3) and (5) the total payment, denoted by $R^{m}(\theta)$, is given by

$$
R^{m}(\theta)=q^{m}(\theta) \theta+t^{m}(q(\theta))=\theta-U^{m}(\theta)
$$

which implies that $\dot{R}(\theta)=1-\dot{U}^{m}(\theta)=q^{m}(\theta) \geq 0$, and that $\dot{R}(\theta)$ is non-increasing in $\theta$ since $\dot{q}^{m}(\theta) \leq 0$. 


\subsection{Proof of Proposition 2}

To find $P_{1}$ 's best response to $P_{2}$ 's contract, we need to solve the following problem, denoted thereafter by $\left(P_{1}^{c}\right)$ :

$$
\max _{\left\{q(\cdot), t_{1}(\cdot)\right\}} \int_{\underline{\theta}}^{\bar{\theta}} f(\theta)\left(-\frac{\tilde{C}(q(\theta))}{2}+\frac{\theta}{2} q(\theta)+t_{1}(q(\theta))\right) d \theta
$$

$$
\text { s.t. (11), (4), (5) and (6). }
$$

It should be clear that problem $\left(\mathcal{P}_{1}^{c}\right)$ is quite similar to problem $\left(\mathcal{P}^{m}\right)$ modulo the fact that $P_{1}$ has now no control on $t_{2}^{c}(\cdot)$. Ignoring (6) for now and proceeding as in the case of the merged principals, we can reduce the problem $\left(\mathcal{P}_{1}^{c}\right)$ to

$$
\max _{q(\cdot)} \int_{\underline{\theta}}^{\bar{\theta}}\left(-\frac{\tilde{C}(q(\theta))}{2}-\frac{\theta q(\theta)}{2}+\theta-t_{2}^{c}(q(\theta))-(1-q(\theta))\left(\frac{1-F(\theta)}{f(\theta)}\right)\right) f(\theta) d \theta .
$$

Let us suppose that $t_{2}^{c}(q)$ is convex in $q$ so that principal 1's problem is concave. We will check ex post that this concavity property is satisfied. Point-wise optimization of this maximand yields the corresponding collective effort that $P_{1}$ would like to implement in a best response to $t_{2}^{c}(\cdot)$ :

$$
\frac{1}{2} \tilde{C}^{\prime}(q(\theta))=-\frac{\theta}{2}-t_{2}^{c \prime}(q(\theta))+\frac{1-F(\theta)}{f(\theta)}
$$

Next we characterize the equilibrium monitoring effort $q(\cdot)$ and argue that we have a symmetric equilibrium in the contracts. Following similar steps as in the case for $P_{1}$, we can indeed derive the best response for $P_{2}$ to a given $t_{1}(\cdot)$, which is symmetric to $P_{1}$ 's best response. Then using the two best responses and the agent's first-order condition (12), we characterize the equilibrium monitoring effort, which is presented in the condition (13):

$$
\tilde{C}^{\prime}\left(q^{c}(\theta)\right)=2\left(\frac{1-F(\theta)}{f(\theta)}\right) .
$$

As in the cooperative case, the monotonicity of the hazard rate implies that $q^{c}(\theta)$ is non-increasing as required by the condition (6).

The principals' best responses imply that the equilibrium transfers are symmetric since the outcome $q^{c}(\theta)$ is unique and $t_{k}^{c}\left(q^{c}(\underline{\theta})\right)=\underline{\theta}\left(1-q^{c}(\underline{\theta})\right)$, for $k=1,2$.

Immediate comparison of (8) with (13) shows that there is excessive monitoring effort when the principals compete in contracts even though monitoring efforts are coordinated in each case:

$$
q^{c}(\theta)>q^{m}(\theta) \quad \text { for all } \theta
$$

except for the highest profit level $\bar{\theta}$ where they are both equal to zero. 
We again see that the total payment is increasing and concave in $\theta$. First, the rent is given by

$$
U^{c}\left(q^{c}(\theta)\right)=\int_{\underline{\theta}}^{\bar{\theta}}\left(1-q^{c}(x)\right) d x,
$$

and from (11) and (5), the total payment, denoted by $R^{c}(\theta)$, is given by

$$
\begin{aligned}
R^{c}(\theta) & =q^{c}(\theta) \theta+t_{1}^{c}(q(\theta))+t_{2}^{c}(q(\theta)) \\
& =\theta-U^{c}(\theta) \\
& =\theta-\int_{\underline{\theta}}^{\theta}\left(1-q^{c}(x)\right) d x
\end{aligned}
$$

The derivative of the total payment with respect to $\theta$ is $q^{c}(\theta) \geq 0$, which is non-increasing in $\theta$. It immediately follows from the comparison of $q^{m}(\theta)$ and $q^{c}(\theta)$ that total payment is higher under centralized monitoring for all $\theta>\underline{\theta}$.

Note also that, in a symmetric equilibrium, we have $t^{c \prime}(q)=-\frac{\theta^{c}(q)}{2}$ where $\theta^{c}(q)$ is the inverse function of $q^{c}(\theta)$. Thus, we get $t^{c \prime \prime}(q)=-\frac{\theta^{c \prime}(q)}{2}>0$ and thus $t^{c}(q)$ is convex validating the concavity of each principal's maximization problem.

\subsection{Proof of Proposition 3}

We will prove the proposition in several stages. In subsection 7.4.1, we begin by characterizing the $S O S C$ as indicated prior to presenting the condition (22). Then we present the principal's problem in subsection 7.4.2, compute the first-order condition and obtain some results, namely: in any symmetric equilibrium (i) $t^{d \prime \prime}(\cdot) \geq 0$, implying that the maximand in (17) is concave as required; and (ii) $\frac{\partial p_{2}^{*}}{\partial p_{1}}<1$, i.e., there is free riding. In subsection 7.4 .3 we characterize the set of symmetric solutions $p^{d}(\theta)$ and show that (i) the ignored conditions (22) are satisfied, (ii) that $p^{c}(\theta)>p^{d}(\theta)$ for $\theta \in(\underline{\theta}, \bar{\theta})$, and (iii) for each solution $p^{d}(\theta)$, there exists a $\hat{\theta}<\bar{\theta}$ such that there will be no monitoring for $\theta>\hat{\theta}$. Then we study more precisely the issue of the corner at zero in subsection 7.4.4, before moving on and checking in subsection 7.4.5 that local incentive constraints imply global incentive compatibility. The issue there is that the "single-crossing property" depends on $p_{2}^{*}(\cdot)$, which is endogenous in this common agency environment, but strategic complementarity of the principals' efforts ensures that we do not encounter any problem. Finally, we demonstrate that the total payment functions are indeed concave as claimed.

\subsubsection{Condition $S O S C$ (22)}

Given the other principal's contract $t_{2}\left(p_{2}\right)$, there is no loss of generality in applying the Revelation Principle to compute $P_{1}$ 's best response in a pure strategy Nash equilibrium between the princi- 
pals. ${ }^{27}$ We will thus construct the best response $\left(t_{1}(\theta), p_{1}(\theta)\right)$ in terms of a direct mechanism. In equilibrium, we will show that $p_{1}(\theta)$ is invertible when $p_{1}(\theta)>0$, using which we will associate to this direct mechanism a nonlinear schedule $t_{1}\left(p_{1}\right)$.

Focusing on the direct mechanism $\left(t_{1}(\theta), p_{1}(\theta)\right)$ for the moment, standard arguments imply that the local $F O C$ for the agent's problem writes as:

$$
\dot{t}_{1}(\theta)+\dot{p}_{1}(\theta) v_{p}\left(\theta, p_{1}(\theta)\right)=0 \quad \text { for all } \theta \text {. }
$$

The corresponding $S O S C$ associated to the agent's problem is given by:

$$
\dot{p}_{1}(\theta)\left[1-p_{2}^{*}\left(p_{1}(\theta), \theta\right)+\left(1-p_{1}(\theta)\right) \frac{\partial p_{2}^{*}}{\partial p_{1}}\left(p_{1}(\theta), \theta\right)\right] \leq 0
$$

which immediately implies $(22)$ since $\frac{\partial p_{2}^{*}}{\partial p_{1}}>0$.

\subsection{2 $P_{1}$ 's problem:}

Let us describe $P_{1}$ 's problem with decentralized monitoring, denoted by $P_{1}^{d}$ :

$$
\begin{gathered}
\max _{\left\{p_{1}(.), t_{1}(.)\right\}} \int_{\underline{\theta}}^{\bar{\theta}} f(\theta)\left[-C\left(p_{1}(\theta)\right)+\frac{\theta}{2}\left(1-\left(1-p_{1}(\theta)\right)\left(1-p_{2}^{*}\left(p_{1}(\theta), \theta\right)\right)\right)+t_{1}\left(p_{1}(\theta)\right)\right] d \theta \quad\left(\mathcal{P}_{1}^{d}\right) \\
\text { s.t. }(4),(17),(18),(20),(21), \text { and }(22) .
\end{gathered}
$$

It is again optimal to set $U(\underline{\theta})=0$, and then from (21), we can integrate to obtain

$$
U(\theta)=\int_{\underline{\theta}}^{\theta}\left(1-p_{1}(x)\right)\left(1-p_{2}^{*}\left(p_{1}, x\right)\right) d x .
$$

We will ignore condition (22) for now and show later that it is indeed satisfied by the equilibrium schedule. After using the indirect utility function (20) to substitute $t_{1}(\cdot)$, we can substitute $U(\theta)$ using (31) to simplify the problem to

$$
\begin{aligned}
& \max _{p_{1}(.)} \int_{\underline{\theta}}^{\bar{\theta}} f(\theta)\left[-C\left(p_{1}(\theta)\right)+\frac{\theta}{2}+\left(1-p_{1}\right)\left(1-p_{2}^{*}\left(p_{1}(\theta), \theta\right)\right)\left(\frac{\theta}{2}-\frac{1-F(\theta)}{f(\theta)}\right)\right. \\
& \left.-t_{2}^{d}\left(p_{2}^{*}\left(p_{1}(\theta), \theta\right)\right)\right] d \theta \\
& \text { s.t. } p_{2}^{*}\left(p_{1}(\theta), \theta\right) \text { satisfies }(18) \text {. }
\end{aligned}
$$

In the remainder of the proof, we suppress $\theta$ where obvious. Assuming concavity of the principal's problem, point-wise optimization yields the following first-order condition for the optimal $p_{1}(\theta)$,

\footnotetext{
${ }^{27}$ See $[24]$ for this argument.
} 
denoted by $p_{1}^{d}(\theta)$, when it is interior (i.e., $p_{1}^{d} \in(0,1)$ ),

$$
\begin{aligned}
C^{\prime}\left(p_{1}^{d}\right)= & \left(1-p_{2}^{*}\left(p_{1}^{d}, \theta\right)\right)\left(\frac{1-F}{f}-\frac{\theta}{2}\right) \\
& -\frac{\partial p_{2}^{*}}{\partial p_{1}}\left(p_{1}^{d}, \theta\right)\left[\left(1-p_{1}^{d}\right)\left(\frac{\theta}{2}-\frac{1-F}{f}\right)+t_{2}^{d \prime}\left(p_{2}^{*}\left(p_{1}^{d}, \theta\right)\right)\right],
\end{aligned}
$$

where $\frac{\partial p_{2}^{*}}{\partial p_{1}}\left(p_{1}^{d}, \theta\right)$ can be obtained from $(18)$ when $p_{2}^{*}\left(p_{1}^{d}, \theta\right)>0$ :

$$
\frac{\partial p_{2}^{*}}{\partial p_{1}}=\frac{\theta}{t_{2}^{d \prime \prime}\left(p_{2}^{*}\left(p_{1}, \theta\right)\right)}
$$

Using this expression, we obtain:

$$
C^{\prime}\left(p_{1}^{d}(\theta)\right)=\left(1-p_{2}^{*}\right)\left(\frac{1-F}{f}-\frac{\theta}{2}\right)+\frac{\theta}{t_{2}^{d \prime \prime}\left(p_{2}^{*}\right)}\left(1-p_{1}\right)\left(\frac{\theta}{2}+\frac{1-F}{f}\right) .
$$

Concavity of the principal's problem is obtained when

$$
-C^{\prime \prime}\left(p_{1}\right)-2 \frac{\partial p_{2}^{*}\left(p_{1}, \theta\right)}{\partial p_{1}}\left(\frac{1-F(\theta)}{f(\theta)}\right)+\frac{\partial^{2} p 2^{*}\left(p_{1}, \theta\right)}{\partial^{2} p_{1}}\left(1-p_{1}\right)\left(\frac{\theta}{2} \frac{1-F(\theta)}{f(\theta)}\right) \leq 0 .
$$

This condition depends on the equilibrium strategy and is thus hard to derive from exogenous parameters. We will thus assume that it is satisfied in what follows.

Let us look for a symmetric equilibrium with $t^{d}(\cdot)=t_{1}^{d}(\cdot)=t_{2}^{d}(\cdot)$ and $p^{d}(\cdot)=p_{1}^{d}(\cdot)=p_{2}^{d}(\cdot)$. For $\theta$ such that $p^{d}(\theta)>0$ and $p_{2}^{*}\left(p_{1}^{d}(\theta) \theta\right)=p^{d}(\theta)$, (34) becomes

$$
C^{\prime}\left(p^{d}\right)=\left(1-p^{d}\right)\left(\frac{1-F}{f}-\frac{\theta}{2}+\frac{\theta}{t^{d \prime \prime}\left(p^{d}\right)}\left(\frac{\theta}{2}+\frac{1-F(\theta)}{f(\theta)}\right)\right) .
$$

Using (18) we have for all $\theta$, where $p^{d}(\theta)>0$,

$$
\theta\left(1-p^{d}(\theta)\right)=-t^{d \prime}\left(p^{d}(\theta)\right)
$$

which after differentiation w.r.t. $\theta$ yields

$$
1-p^{d}-\theta \dot{p}^{d}=-t^{d \prime \prime}\left(p^{d}\right) \dot{p}^{d},
$$

or

$$
\frac{\theta}{t^{d \prime \prime}\left(p^{d}\right)}=-\frac{\theta \dot{p}^{d}}{1-p^{d}-\dot{p}^{d} \theta} .
$$

Since $\dot{p}^{d}(\theta) \leq 0$, as required by the local $\operatorname{SOSC}(22)$, the above expression establishes two results: (i) $t^{d \prime \prime}(\cdot) \geq 0$, and using (33), (ii) $\frac{\partial p_{2}^{*}}{\partial p_{1}}<1$.

Hence, using (38) to replace $t^{d \prime \prime}(\cdot)$ in (36) yields the following differential equation satisfied by a symmetric equilibrium at all points $\theta$ where $p^{d}(\theta)>0$.

$$
C^{\prime}\left(p^{d}\right)=\left(1-p^{d}\right)\left(\frac{1-F}{f}-\frac{\theta}{2}-\frac{\theta \dot{p}^{d}}{1-p^{d}-\dot{p}^{d} \theta}\left(\frac{\theta}{2}+\frac{1-F}{f}\right)\right) .
$$


A priori, the solution to this differential equation, denoted by $s(\theta)$, does not need to remain positive (as is required for $p^{d}(\theta)$ ). Hence we use the different notation to distinguish $s(\theta)$ from the equilibrium schedule $p^{d}(\theta)$. We intend to prove that $p^{d}(\theta)=\max \{s(\theta), 0\}$. To define the solutions to this differential equation for all values of $\theta$, we will extend $C(s)$ for $s<0$. The continuously differentiable extension $C(s)=0$ for $s<0$ does the job. Once a solution, denoted by $s(\theta)$, to this differential equation is obtained, it cannot be positive again once it touches zero since it is weakly decreasing. We then have to check that $p^{d}(\theta)=\max \{s(\theta), 0\}$, i.e., there is a corner at zero in the optimum of the principal's objective.

First, note that (39) can be also written as

$$
\frac{\theta \dot{s}^{d}}{1-s}=\frac{(1-s)\left(\frac{1-F}{f}-\frac{\theta}{2}\right)-C^{\prime}(s)}{2(1-s)\left(\frac{1-F}{f}\right)-C^{\prime}(s)} .
$$

Let us now analyze the properties of $s(\theta)$, the solutions to (40).

Note first that the initial value $s(\underline{\theta})$ is not defined a priori. The initial value $s(\underline{\theta})$ is thus chosen to guarantee that the whole schedule $s(\theta)$ satisfies the local SOSC.

\subsubsection{Comparative Statics}

See Figure 2 for this. Let us note that the numerator (resp. denominator) of the $R H S$ above is equal to zero for $p_{L}(\theta)$ (resp. $p^{c}(\theta)$ ), where $p_{L}(\theta)$ is defined in $(25)$. Note that $p^{c}(\theta)$ is defined for all $\theta \in[\underline{\theta}, \bar{\theta}]$. Instead, $p_{L}(\theta)$ is strictly positive only for $\theta \in\left[\underline{\theta}, \theta^{*}\right]$, where $\theta^{*}$ is defined by (15), and there exists a unique $\theta^{*} \in(\underline{\theta}, \bar{\theta})$. Both $p^{c}(\theta)$ and $p_{L}(\theta)$ are decreasing with $\theta$ and $p^{c}(\theta)>p_{L}(\theta)$ for all $\theta \in[\underline{\theta}, \bar{\theta})$. From $(40), s(\theta)$ is non-increasing as long as $s(\theta)$ remains in the interval $\left[p_{L}(\theta), p^{c}(\theta)\right]$.

Let us take $s(\underline{\theta}) \in\left[p_{L}(\underline{\theta}), p^{c}(\underline{\theta})\right]$ and consider the solution to (40) with this initial value. We claim that such a solution is strictly decreasing and always remains within $\left(p_{L}(\theta), p^{c}(\theta)\right)$. Indeed, if it crosses $p_{L}(\theta)$ at any $\theta_{1} \in\left(\underline{\theta}, \theta^{*}\right)$ for the first time, we should have $\dot{s}\left(\theta_{1}\right)=0$, but then $s(\theta)<p_{L}(\theta)$ for $\theta \in\left(\theta_{1}-\varepsilon, \theta_{1}\right)$ for $\varepsilon$ small enough; a contradiction.

If $s(\theta)$ crosses $p^{c}(\theta)$ at any $\theta_{2} \in(\underline{\theta}, \bar{\theta})$ for the first time, we should have $\dot{s}\left(\theta_{2}\right)=\infty$, but then $s(\theta)>p^{c}(\theta)$ for $\theta_{2} \in\left(\theta_{2}, \theta_{2}+\varepsilon\right)$ for $\varepsilon$ small enough; a contradiction since the trajectories do not go through $\bar{\theta}$.

Since all initial values $s(\underline{\theta})$ in $\left[p_{L}(\underline{\theta}), p^{c}(\underline{\theta})\right]$ correspond to possible solutions to (40), all these solutions are equilibria as long as $s(\theta) \geq 0$, i.e., are on an interval $[\underline{\theta}, \hat{\theta}]$, where $\hat{\theta}$ is defined by $s(\hat{\theta})=0$ for any given $s(\cdot)$ and $\hat{\theta}<\bar{\theta}$. These schedules correspond to equilibria which can be ranked from low to high depending on their initial value $p^{d}(\underline{\theta})$. 


\subsubsection{Corner at zero}

Let us now tackle more precisely the issue of the corner at zero. So far indeed, we have implicitly assumed that $P_{1}$ only induces the agent, whatever his profit level $\theta$, to choose a positive monitoring intensity $p_{2}^{*}\left(p_{1}, \theta\right)$ from $P_{2}$. We must investigate what happens when $P_{1}$ chooses to induce $p_{2}^{*}\left(p_{1}, \theta\right)=0$.

Indeed, take any supposed equilibrium $p^{d}(\theta)$. For $\theta>\hat{\theta}$ we have $p^{d}(\theta)=0$ and when, say $P_{1}$, deviates and makes an alternative offer to the common agent, he may want to have the agent choose $p_{2}^{*}\left(p_{1}, \theta\right)=0$. Note that this occurs when

$$
-\theta\left(1-p_{1}\right) \leq t^{d \prime}(0)=-\hat{\theta}
$$

where the equality follows from the definition of $\hat{\theta}$ and continuity of $t^{d^{\prime}}(\cdot)$ at $\hat{\theta}$. Hence, such deviation occurs as long as $p_{1} \leq 1-\frac{\hat{\theta}}{\theta}$. For $\theta<\hat{\theta}$, there is no such deviation with $p_{1}>0$ inducing the agent to choose $p_{2}^{*}\left(p_{1}, \theta\right)=0$. For $\theta \geq \hat{\theta}$ instead, there are such deviations. Over the interval of $p_{1}$ in $\left[\underline{\theta}, 1-\frac{\hat{\theta}}{\theta}\right], P_{1}$ 's marginal payoff at $\theta$ is

$$
-C^{\prime}\left(p_{1}\right)+\frac{1-F}{f}-\frac{\theta}{2}<0
$$

because $\theta \geq \hat{\theta} \geq \theta^{*}$. Hence, $P_{1}$ 's optimal choice on the set is $p_{1}=0$.

For $p_{1}$ in the interval $\left[1-\frac{\hat{\theta}}{\theta}, 1\right], P_{1}$ 's marginal payoff at $\theta$ is

$$
-C^{\prime}\left(p_{1}\right)+\left(1-p_{2}^{*}\left(p_{1}, \theta\right)\right)\left(\frac{1-F}{f}-\frac{\theta}{2}\right)-\frac{\partial p_{2}^{*}}{\partial p_{1}}\left(p_{1}, \theta\right)\left(\left(1-p_{1}\right)\left(\frac{\theta}{2}-\frac{1-F}{f}\right)+t^{d \prime}\left(p_{2}^{*}\left(p_{1}, \theta\right)\right) .\right.
$$

We know that this expression is zero at $p_{1}=s(\theta)$. Thus, by concavity of $P_{1}$ 's payoff, the expression above is negative at $p_{1}=1-\frac{\hat{\theta}}{\theta}$. Since $P_{1}$ 's payoff is continuous at $p_{1}=1-\frac{\hat{\theta}}{\theta}$, we can deduce that $P_{1}$ 's payoff is maximum at $p_{1}=0$ for all $\theta \geq \hat{\theta}$.

\subsubsection{Global concavity}

To ensure global concavity of the agent's problem, let us write

$$
\Delta=v\left(p^{d}(\theta), \theta\right)-t^{d}(\theta)-\left[v\left(p^{d}\left(\theta_{1}\right), \theta\right)-t^{d}\left(\theta_{1}\right)\right],
$$

where

$$
v\left(p^{d}\left(\theta_{1}\right), \theta\right)=\max _{p_{2}}\left\{\theta\left(1-p^{d}\left(\theta_{1}\right)\right)\left(1-p_{2}\right)-t^{d}\left(p_{2}\right)\right\}
$$

and let us show that $\Delta \geq 0$ for all $\left(\theta, \theta_{1}\right)$ in $[\underline{\theta}, \bar{\theta}] \times[\underline{\theta}, \bar{\theta}]$.

Using the $F O C$ of the agent's problem (30), we have: 


$$
\begin{aligned}
\Delta & =v\left(p^{d}(\theta), \theta\right)-v\left(p^{d}\left(\theta_{1}\right), \theta\right)-\int_{\theta_{1}}^{\theta} v_{p}\left(p^{d}(y), y\right) \dot{p}(y) d y \\
& =\int_{\theta_{1}}^{\theta}\left[v_{p}\left(p^{d}(y), \theta\right)-v_{p}\left(p^{d}(y), y\right)\right] \dot{p}(y) d y \\
& =\int_{\theta_{1}}^{\theta} \dot{p}(y)\left(\int_{y}^{\theta} v_{p \theta}\left(p^{d}(y), x\right) d x\right) d y .
\end{aligned}
$$

Since $\dot{p}(y) \leq 0, \Delta \geq 0$ when $v_{p \theta}\left(p^{d}(y), x\right) \leq 0$. But, by definition,

$$
\begin{aligned}
v_{p \theta}\left(p^{d}(y), x\right) & =-\left(\left(1-p^{d}(y)\right) \frac{\partial p_{2}^{*}}{\partial p_{1}}\left(p^{d}(y), x\right)+1-p_{2}^{*}\left(p^{d}(y), x\right)\right) \\
& \leq 0
\end{aligned}
$$

since $\frac{\partial p_{2}^{*}}{\partial p_{1}}\left(p^{d}(y), x\right) \geq 0$.

\subsubsection{Concavity of total payment and comparison with centralized case}

First, observe that total payment, denoted by $R^{d}(\theta)$ is given by

$$
\begin{aligned}
R^{d}(\theta) & =\left[2 p_{i}^{d}(\theta)-\left(p_{i}^{d}(\theta)\right)^{2}\right] \theta+t^{d}\left(p_{i}^{d}(\theta)\right)+t^{d}\left(p_{i}^{d}(\theta)\right) \\
& =\theta-U^{d}(\theta) .
\end{aligned}
$$

From (21) the derivative of $R^{d}(\theta)$ is:

$$
\left[1-\left(1-p^{d}(\theta)\right)^{2}\right]>0
$$

but then the second derivative is $2\left(1-p^{d}(\theta)\right) \dot{p}^{d}(\theta) \leq 0$ as is required for concavity. Comparing equilibrium monitoring efforts we immediately see that total payment is smaller under decentralized monitoring compared to the centralized case for all $\theta>\underline{\theta}$. This is depicted in Figure 2.

\subsection{Proof of Proposition 4}

To prove both parts of the proposition, we only need to show that the trajectories of the differential equation (39), that characterizes $p^{d}(\theta)$, may cross the line $p=p^{m}(\theta)$ only once from above, where $p^{m}(\theta)$ is given by (10). We have already argued in subsection 7.4 .3 that $p^{d}(\theta)$ cannot intersect $p^{c}(\theta)$ from below, and thus we also have $\tilde{\theta}<\bar{\theta}$.

Denoting an intersection point of $p^{d}(\theta)$ and $p^{m}(\theta)$ as $\theta_{1}$, where $p^{d}\left(\theta_{1}\right)=p^{m}\left(\theta_{1}\right)$, we have from (40):

$$
\frac{\dot{p}^{d}\left(\theta_{1}\right)}{1-p^{d}\left(\theta_{1}\right)}=-\frac{1}{2 \frac{1-F\left(\theta_{1}\right)}{f\left(\theta_{1}\right)}}
$$


since $C^{\prime}\left(p^{d}\left(\theta_{1}\right)\right)=\left(1-p^{d}\left(\theta_{1}\right)\right)\left(\frac{1-F\left(\theta_{1}\right)}{f\left(\theta_{1}\right)}\right)$ by $(10)$ when $p^{d}\left(\theta_{1}\right)=p^{m}\left(\theta_{1}\right)$.

Also by differentiating (10), we get:

$$
\frac{\dot{p}^{m}\left(\theta_{1}\right)}{1-p^{m}\left(\theta_{1}\right)}=\frac{\left.\frac{d}{d \theta}\left(\frac{1-F}{f}\right)\right|_{\theta_{1}}}{C^{\prime \prime}\left(p^{m}\left(\theta_{1}\right)\right)+\frac{1-F\left(\theta_{1}\right)}{f\left(\theta_{1}\right)}} .
$$

Comparing (41) and (42) at a point $\theta_{1}$ such that $p^{m}\left(\theta_{1}\right)=p^{d}\left(\theta_{1}\right)$, we observe that $0>$ $\dot{p}^{m}\left(\theta_{1}\right)>\dot{p}^{d}\left(\theta_{1}\right)$ when $\frac{d}{d \theta}\left(\frac{1-F}{f}\right) \geq-\frac{1}{2}$.

Therefore, any trajectory $p^{d}(\theta)$ starting below $p^{m}(\theta)$ stays below $p^{m}(\theta)$ and those starting above $p^{m}(\theta)$ must intersect $p^{d}(\theta)$ once from above at some $\tilde{\theta}<\bar{\theta}$ and then stays below $p^{m}(\theta)$.

\subsection{Comparison of profits between centralized and decentralized monitoring}

The comparison between the two non-cooperative modes is made quite difficult because it requires first to compute explicitly the equilibrium schedule $p^{d}(\theta)$ which is a solution to a highly nonlinear differential equation and, if such a solution can be characterized, to compute the expected overall cost of the corresponding allocation. To nevertheless give an example of such a profit comparison between the two non-cooperative institutional arrangements, consider the case of an exponential distribution on $[\underline{\theta},+\infty], F(\theta)=1-e^{-2(\theta-\underline{\theta})}$, with $0<\underline{\theta}<1$ to guarantee that a positive $\theta^{*}<1$ such that $p_{L}\left(\theta^{*}\right)=0$. Of course, this distribution has an unbounded support, but apart from the fact that there is 'distortion at the top' both under the merged and the centralized cases, all our previous results go through. Assume that the cost function is now given by $C(p)=\frac{p^{2}}{2}$ on $\left[0, \frac{1}{2}\right]$ and $\infty$ otherwise so that the condition of Lemma 1 holds.

With these functional forms, the overall cost of extracting income using a monitoring schedule $p(\theta)$ can be written as follows:

$$
\begin{aligned}
\omega(p) & =\int_{\underline{\theta}}^{\infty}\left(2 C(p(\theta))+\frac{1-F(\theta)}{f(\theta)}(1-p(\theta))^{2}\right) f(\theta) d \theta \\
& =\int_{\underline{\theta}}^{\infty}\left(p^{2}(\theta)+\frac{(1-p(\theta))^{2}}{2}\right) 2 e^{-2(\theta-\underline{\theta})} d \theta
\end{aligned}
$$

where the hazard rate $\frac{1-F(\theta)}{f(\theta)}=\frac{1}{2}$ for the exponential distribution. Point-wise minimization of expression (43) yields the cooperative solution,

$$
C^{\prime}\left(p^{m}(\theta)\right)=\frac{1}{2}\left(1-p^{m}(\theta)\right), \text { for all } \theta
$$

which for this example is $p^{m}(\theta)=\frac{1}{3}$, and yields an expected $\operatorname{cost} \omega\left(p^{m}\right)=\frac{1}{3}$.

From (13), under centralized monitoring, the monitoring intensity by either principal is given by

$$
C^{\prime}\left(p^{c}(\theta)\right)=1-p^{c}(\theta), \text { for all } \theta \geq \underline{\theta},
$$


i.e., $p^{c}(\theta)=\frac{1}{2}$, with an expected cost $\omega\left(p^{c}\right)=\frac{3}{8}$ which is of course greater than $\frac{1}{3}$.

From (40) in the Appendix, under decentralized monitoring, an equilibrium schedule $p^{d}(\theta) \geq 0$ solves the following differential equation:

$$
\frac{\theta \dot{p}^{d}(\theta)}{1-p^{d}(\theta)}=\frac{\frac{1-\theta}{2}-\frac{p^{d}(\theta)}{1-p^{d}(\theta)}}{1-\frac{p^{d}(\theta)}{1-p^{d}(\theta)}}
$$

with the initial conditions $p^{d}(\underline{\theta})$ in $\left[p_{L}(\underline{\theta}), \frac{1}{2}\right]$, where $p_{L}(\theta)$ is obtained as in subsection 7.4 .3 by setting the numerator to zero on the right hand side of the above expression. We therefore have,

$$
p_{L}(\theta)=\max \left\{0, \frac{1-\theta}{3-\theta}\right\} .
$$

The nonlinearity of this differential equation makes it impossible to solve explicitly for the equilibrium schedules. Therefore, we use numerical methods to compute the solution $p^{d}(\theta)$ for $\underline{\theta}=0.0001$ and $p^{d}(\underline{\theta})=\frac{1}{3}$. Then we use this $p^{d}(\theta)$ to calculate the implementation cost to show that $w\left(p^{*}\right)$ is strictly smaller than $\omega\left(p^{c}\right)=\frac{3}{8} \cdot{ }^{28}$ Of course, this strict inequality still holds for $\underline{\theta}$ small enough for any equilibrium under decentralized monitoring which has an initial value $p^{d}(\underline{\theta})$ in $\left[p_{L}(\underline{\theta}), \frac{1}{3}\right]$. Decentralized monitoring is thus preferred for a distribution of profits putting enough weight on low levels of profit.

Let us turn to the case where $\underline{\theta}$ is close to 1 from below. We then have $\theta^{*}$ close to $\underline{\theta}$ and $p_{L}(\theta)$ close to 0 everywhere. Take an equilibrium schedule $p^{d}(\theta)$ under decentralized monitoring with an initial value $p^{d}(\underline{\theta})$ close to $p_{L}(\underline{\theta})$. Such solution remains close to zero everywhere and thus $\omega\left(p^{d}\right) \approx \frac{1}{2}>\omega\left(p^{c}\right)$. Centralized monitoring performs better than the decentralized arrangement. The intuition is now the reverse image of the above case; as the distribution of profit puts enough weight on higher levels of profit, centralized monitoring comes closer to the cooperative outcome.

Even with this simple example, the overall picture is quite mixed although it reflects our initial intuition. First, there is incentive to monitor too much in the centralized case, and to monitor too little in the decentralized case due to free riding; second, the former effect is more relevant for low profits while the latter dominates for high profits. We constructed an example to show that if the distribution of profits puts enough weight on low profits, decentralized monitoring will be preferred by the principals, while the opposite is true if the distribution puts enough weight on high profits. In general, comparisons between the two non-cooperative arrangements depend in subtle ways on the profit distribution.

\footnotetext{
${ }^{28}$ The calculated $w\left(p^{*}\right)$ is smaller than 0.35563 . It was derived using the software Mathcad and the details are available from the authors upon request.
} 


\section{References}

[1] B.D. Bernheim, M.D. Whinston, Common marketing agency as a device for facilitating collusion, RAND J. Econ. 16 (1985), 269-281.

[2] B.D. Bernheim, M.D. Whinston, Common agency, Econometrica 54 (1986), 923-943.

[3] B. Biais, D. Martimort, J-C. Rochet, Competing mechanisms in a common value environment, Econometrica 68 (2000), 799-838.

[4] D.S. Bizer, P.M. DeMarzo, Sequential banking, J. Polit. Economy 100 (1992), 41-61.

[5] P. Bolton, D.S. Scharfstein, Optimal debt structure and the optimal number of creditors, J. Polit. Economy 104 (1996), 1-25.

[6] K. Border, J. Sobel, Samurai accountant: a theory of auditing and plunder, Rev. Econ. Stud. 54 (1987), 525-540.

[7] J.H. Boyd, B.D. Smith, The use of debt and equity in the optimal financial contracts, J. Finan. Intermediation 8 (1999), 270-316.

[8] E. Carletti, The structure of bank relationship, endogenous monitoring, and loan rates, J. Finan. Intermediation 13 (2004), 58-86.

[9] E. Detragiache, P. Garella, L. Guiso, Multiple versus single banking relationships: theory and evidence, J. Finance 55 (2000), 1385-1403.

[10] M. Dewatripont, E. Maskin, Credit and efficiency in centralized and decentralized economies, Rev. Econ. Stud. 60 (1995), 35-52.

[11] D. Diamond, Financial intermediation and delegated monitoring, Rev. Econ. Stud. 51 (1984), 393-414.

[12] X. Freixas, J-C. Rochet, Microeconomics of Banking, MIT Press, Cambridge, Mass., 1997.

[13] D. Gale, M. Hellwig, Incentive-compatible debt contracts: the one-period problem, Rev. Econ. Stud. 52 (1985), 647-63.

[14] M. Harris, A. Raviv, The theory of capital structure, J. Finance 46 (1991), 297-355.

[15] O. Hart, Firms, Contracts, and Financial Structure, Oxford Univ. Press, Oxford, 1995.

[16] M. Hellwig, Banking, financial intermediation and corporate finance, in: A. Giovannini, C. Mayer (Eds.) European Financial Integration, Cambridge Univ. Press, Cambridge, (1991), pp. 35-63. 
[17] S. Krasa, A. Villamil, Optimal contracts when enforcement is a decision variable, Econometrica 68 (2000), 119-134.

[18] F. Khalil, B.M. Parigi, The loan size as a commitment device, Int. Econ. Rev. 39 (1998), 135-150.

[19] J.J. Laffont, J. Tirole, Privatization and incentives, J. Law, Econ., Organ. 6 (1991), 1-32.

[20] R. La Porta, F. Lopez-de-Silanes, A. Shleifer, R. Vishny, Law and finance, J. Polit. Economy 106 (1998), 1113-1155.

[21] R. La Porta, F. Lopez-de-Silanes, A. Shleifer, R. Vishny, Investor protection and corporate valuation, J. Finance 57 (2002), 1147-1170.

[22] D. Martimort, Multi-pricipaux avec anti-selection, Ann. Econ. Statist. 28 (1992), 1-38.

[23] D. Martimort, Exclusive dealing, common agency, and multiprincipals incentive theory, RAND J. Econ. 27 (1996), 1-31.

[24] D. Martimort, L. Stole, The revelation and delegation principles in common agency games, Econometrica 70 (2002), 1659-1674.

[25] D. Martimort, L. Stole, Contractual externalities and common agency equilibria, Advances Theoretical Econ. 3 (2003), Article 4.

[26] C. Mezzetti, Common agency with horizontally differentiated principals, RAND J. Econ. 28 (1997), 323-345.

[27] M. Pagano, A. Röell, The choice of stock ownership structure: agency costs, monitoring, and the decision to go public, Quart. J. Econ. 113 (1998), 187-226.

[28] C. Parlour, U. Rajan, Competition in loan contracts, Amer. Econ. Rev. 91 (2001), 187-226.

[29] M. Peters, Common agency and the revelation principle, Econometrica, 69 (2001), 1349-72.

[30] M. Peters, Negotiation and take-it-or-leave-it in common agency, J. Econ. Theory 111 (2003), 88-109.

[31] R. Rajan, Insiders and outsiders: the choice between informed and arm's length debt, J. Finance 47 (1992), 1367-1400.

[32] J-C. Rochet, The taxation principle and multitime Hamilton-Jacobi equations, J. Math. Econ. 14 (1985), 113-128. 
[33] I. Segal, Contracting with externalities, Quart. J. Econ. 114 (1999), 337-389.

[34] A. Shleifer, R. Vishny, Large shareholders and corporate control, J. Polit. Economy 94 (1986), $461-88$.

[35] A. Shleifer, D. Wolfenzon, Investor protection and equity markets, J. Finan. Econ. 66 (2002), $3-27$.

[36] L. Stole, Mechanism design under common agency: theory and applications, working paper, University of Chicago, GSB, (1997).

[37] J. Tirole, Corporate governance, Econometrica 69 (2001), 1-35.

[38] J. Tirole, Financial Crises, Liquidity, and the International Monetary System, Princeton Univ. Press, Princeton, NJ, 2002.

[39] R. Townsend, Optimal contracts and competitive markets with costly state verification, J. Econ. Theory 21 (1979), 265-93.

[40] A. Winton, Costly state verification and multiple investors: the role of seniority, Rev. Finan. Stud. 8 (1995), 91-123. 


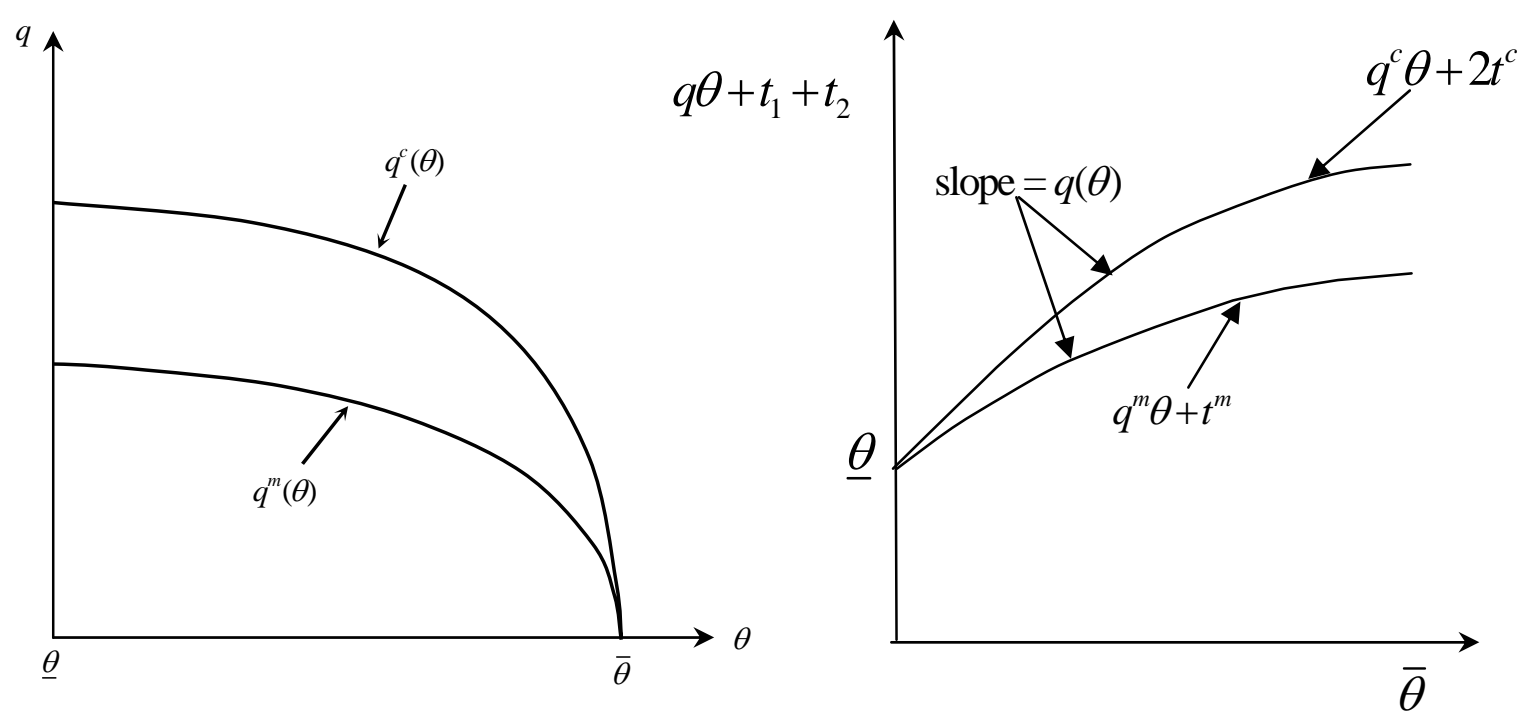

Figure 1. Comparison of monitoring and total payment under cooperative (merged) and non-cooperative but centralized monitoring

$q \theta+t_{1}+t_{2}$
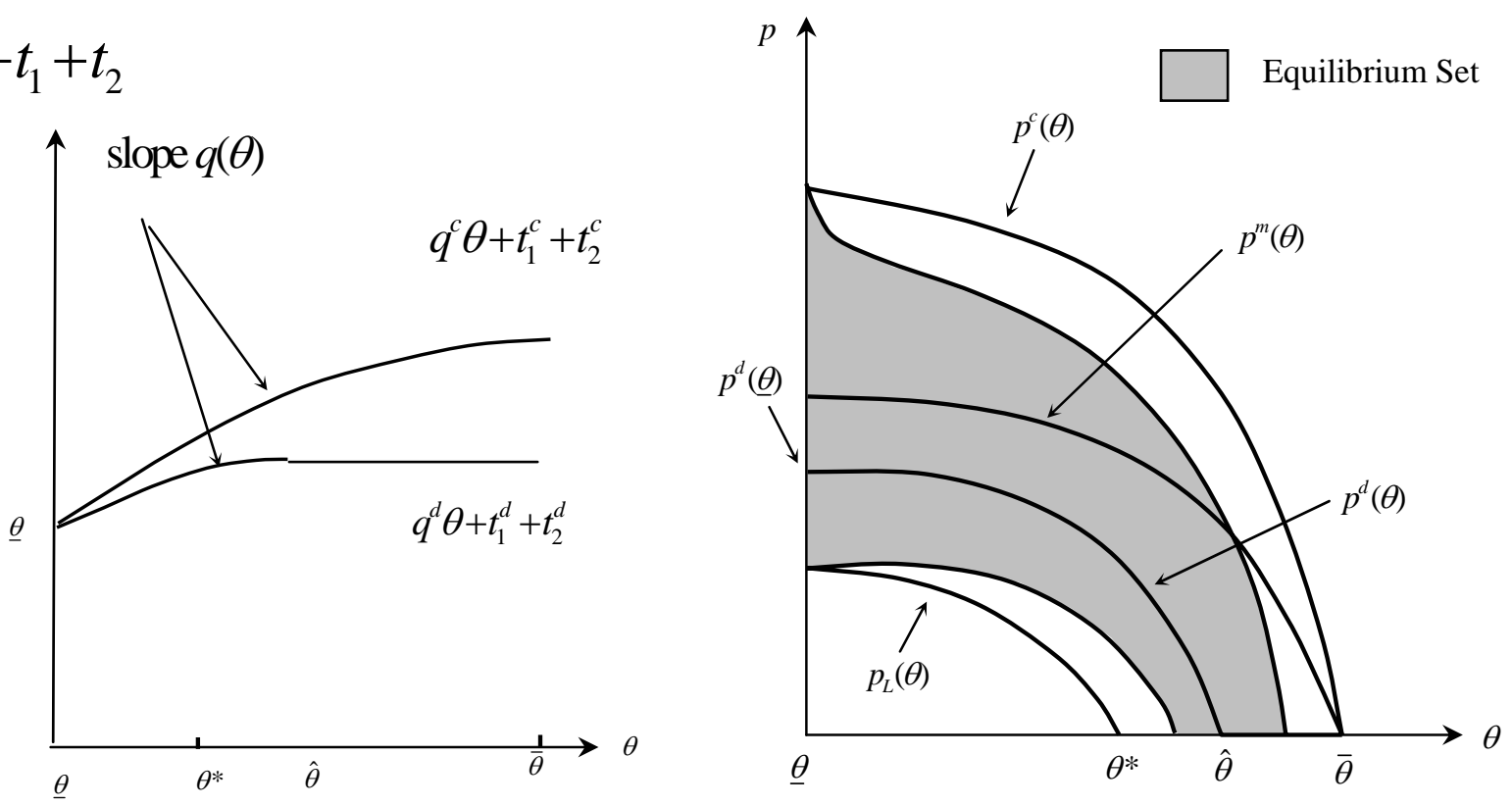

Figure 2. Comparison of total payment under centralized and decentralized monitoring and the set of equilibria under decentralized monitoring. 